\title{
Black Holes at the LHC
}

Panagiota Kanti

\begin{abstract}
In these two lectures, we will address the topic of the creation of small black holes during particle collisions in a ground-based accelerator, such as LHC, in the context of a higher-dimensional theory. We will cover the main assumptions, criteria and estimates for their creation, and we will discuss their properties after their formation. The most important observable effect associated with their creation is likely to be the emission of Hawking radiation during their evaporation process. After presenting the mathematical formalism for its study, we will review the current results for the emission of particles both on the brane and in the bulk. We will finish with a discussion of the methodology that will be used to study these spectra, and the observable signatures that will help us identify the black-hole events.
\end{abstract}

\section{Introduction}

These two lectures aim at offering an introduction to the idea that miniature black holes may be created during high-energy particle collisions at ground-based colliders. This scenario can only be realised in the context of higher-dimensional theories, i.e. theories that postulate the existence of additional spacelike dimensions in nature. An introduction to the two most important versions of these theories, namely the scenario with Large Extra Dimensions and the one with Warped Extra Dimensions will be our starting point.

We will then proceed to introduce the idea of the possible creation of black holes at the laboratory. We will present some simple but illuminating geometrical criteria for this to happen. We will then discuss the boundary value problem whose solution determines whether a black hole has been formed out of two colliding particles. Certain aspects of the creation process will be studied in more detail, namely the amount

Panagiota Kanti

Division of Theoretical Physics, Physics Department, University of Ioannina, Ioannina GR-45110, Greece. e-mail:pkanti@cc.uoi.gr 
of energy that is absorbed by the created black hole and the value of the production cross-section. We will finally discuss the properties of the produced black holes, such as the horizon value, temperature and lifetime, and compare with the ones of their 4-dimensional analogues. The non-vanishing, in general, temperature of the black hole is associated with the emission of a thermal type of radiation from the black hole, i.e. the Hawking radiation. This has its source at the creation of a virtual pair of particles outside the horizon of the black hole (or, equivalently, the quantum tunneling of a particle from within the black hole horizon). We will finish our first lecture with a brief outline of the mathematical formalism that was developed for the study of the Hawking radiation.

The emission of Hawking radiation, i.e. of elementary particles with a thermal spectrum, takes place during the two intermediate phases in the life of a black hole. These are the spin-down phase and the Schwarzschild phase, in chronological order. Starting from the second, that has the simplest gravitational background, we will present a review of the results that have been derived in the literature related to the form of the radiation spectra and their most characteristic features, including their dependence on the dimensionality of spacetime and the relative emissivities of different species of fields. A similar task will then be taken for the spin-down phase during which the black hole carries a non-vanishing angular momentum. In this case, the radiation spectra will have an extra dependence on the angular momentum parameter of the black hole, as well as an angular distribution in space due to the existence of a preferred direction in space, that of the rotation axis. The most important part, from the phenomenological point of view, will be the emission of the black hole directly on the brane on which the Standard Model particles and the observers themselves are located. However, the bulk emission will also be considered as this will determine the amount of energy remaining for emission on the brane.

Having completed the theoretical study of the radiation spectra from a higherdimensional black hole, we now need to address the question of what information we may deduce from these spectra, if one day we manage to detect them, and in what way. Certain properties of the produced black hole such as the mass and temperature need to be determined first. From these, one may then turn to the derivation of more fundamental parameters such as the dimensionality of the gravitational background, or even the value of the fundamental Planck scale and the cosmological constant. As we will see, this task is highly non-trivial and demands the close cooperation of theoretical studies and experimental skill. But if it works, it might provide answers to the most fundamental questions in theoretical physics.

\section{First Lecture: Creation of Black Holes and their Properties}

During the first lecture, we will set the stage for the production and subsequent detection of higher-dimensional black holes. After a brief introduction to models with extra dimensions, we will discuss the possibility of the creation of a black hole during a particle collision, and address certain questions related to this phenomenon. 
We will then turn to the evaporation process of the black hole, and we will briefly present the mathematical formalism for the study of the Hawking radiation.

\subsection{Extra Dimensions}

It is an amazing feature of the Theory of General Relativity that it can be straightforwardly extended to an arbitrary number of dimensions. Its main mathematical construction, Einstein's field equations

$$
G_{\mu v}=R_{\mu \nu}-\frac{1}{2} g_{\mu \nu} R=\kappa^{2} T_{\mu \nu}
$$

are expressed in terms of second-rank tensors whose indices can take any values, depending on our assumptions for the dimensionality of spacetime, without its mathematical consistency to be in any danger. It comes therefore as no surprise that, only a few years after Einstein formulated his theory of gravity, Kaluza produced a gravitational model in five dimensions. The model was soon supplemented with further suggestions about the topology of the extra dimension by Klein, and it was the first attempt ever to derive a unification theory in which gravity played the fundamental role.

Klein pictured the extra spacelike dimension introduced by Kaluza as a regular, compact one with finite size $\mathscr{R}$. To avoid any conflicts with observational data, the size of the extra dimension was assumed to be much smaller than any observable length scale. The idea was extensively used decades later in the formulation of String Theory: there, the size of the additional six spacelike dimensions, necessary for the mathematical and physical consistency of the theory, was assumed to be $\mathscr{R}=l_{P}=$ $10^{-33} \mathrm{~cm}$. However, all traditional ideas about the structure, size and use of the extra space radically changed in the 90's. The start was made in the context of string theory, where the idea [1, 2, 3] that the string scale does not necessarily need to be tied to the Planck scale, $M_{P} \simeq 10^{19} \mathrm{GeV}$, was put forward. This soon led 1 to the construction of two, much simpler but extremely rich from the phenomenological point of view, gravitational models: the scenario with Large Extra Dimensions [8, 9] and the one with Warped Extra Dimensions [10].

The topological structure of the higher-dimensional spacetime in each case is shown in Figs. 1 $a, b)$. In the scenario with Large Extra Dimensions, depicted in Fig. 1 a), a 4-dimensional brane is embedded in a $(4+n)$-dimensional flat space with $(3+1)$ non-compact and $n$ spacelike compact dimensions. All ordinary matter, made up of and interacting through Standard Model (SM) fields, is localised on the brane, and experiences gravitational forces that become strong at Planck scale. On the other hand, gravitons, and possibly scalars or other fields not carrying any charges under the SM gauge group, can propagate in the full spacetime. The higher-dimensional,

\footnotetext{
${ }^{1}$ For some early attempts to construct higher-dimensional gravitational models, see Refs. $4,5,6$ 7].
} 


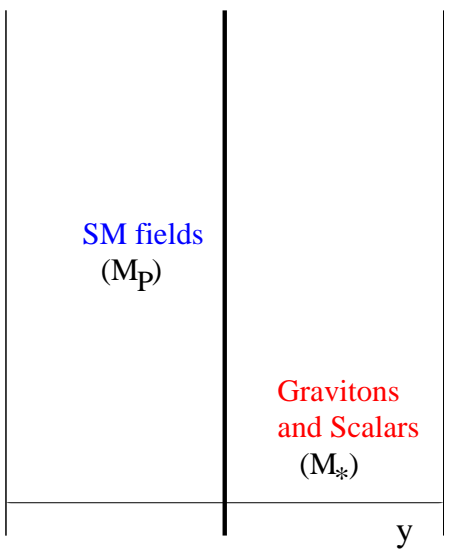

(a)

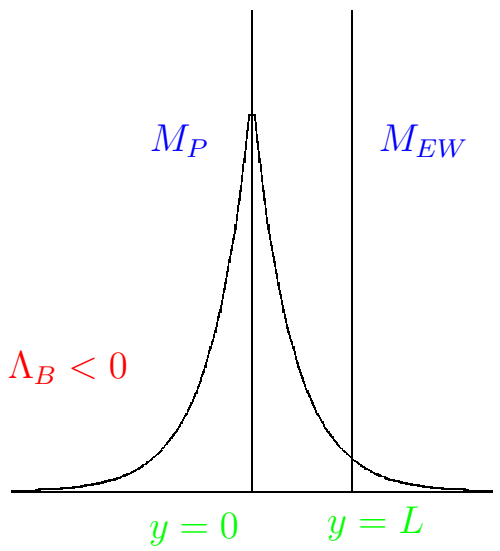

(b)

Fig. 1 (a) A 3-brane embedded in a $(4+n)$-dimensional flat spacetime. (b) Two 3-branes embedded in a 5-dimensional Anti de Sitter spacetime.

fundamental theory has a new scale for gravity, $M_{*}$, that is related to the effective 4-dimensional one through the equation [8, 9]

$$
M_{P}^{2} \simeq \mathscr{R}^{n} M_{*}^{2+n} .
$$

According to the above, if $\mathscr{R} \gg l_{P}$, the fundamental scale for gravity $M_{*}$ can be significantly lower than the 4-dimensional one. By inverting the above relation and using the definition $G_{D}=1 / M_{*}^{n+2}$ for the fundamental gravitational constant, we find

$$
G_{4} \mathscr{R}^{n} \simeq G_{D}
$$

This means that, while, for $r \gg \mathscr{R}$, the Newtonian potential between two masses $m_{1}$ and $m_{2}$ is given by the well-known 4-dimensional formula

$$
V(r)=G_{4} \frac{m_{1} m_{2}}{r},
$$

for $r \ll \mathscr{R}$, the corresponding potential is now not only a higher-dimensional one but a much stronger one for the same masses $m_{1}$ and $m_{2}$, and is written as

$$
V(r)=G_{D} \frac{m_{1} m_{2}}{r^{n+1}} .
$$

In the case of the scenario with Warped Extra Dimensions, shown in Fig. 11 b), a 4-dimensional brane is embedded in the higher-dimensional spacetime which now is five-dimensional. The extra spacelike dimension is generically non-compact but it may be compactified at will if a second brane is introduced in the model. The visible brane, where all SM fields live, is placed at a finite distance $y=L$ from the hidden brane located at $y=0$. If all fundamental scales at the hidden brane are of the order 
Table 1 Current limits on the fundamental energy scale

\begin{tabular}{lrr}
\hline Type of Experiment/Analysis & $M_{*} \geq$ & $M_{*} \geq$ \\
\hline $\begin{array}{l}\text { Collider limits on the production } \\
\text { of real or virtual KK gravitons [11]-[13] }\end{array}$ & $1.45 \mathrm{TeV}(n=2)$ & $0.6 \mathrm{TeV}(n=6)$ \\
Torsion-balance Experiments[14][15] & $3.2 \mathrm{TeV}(n=2)$ & $(\mathscr{R} \leq 50 \mu \mathrm{m})$ \\
Overclosure of the Universe[16] & $8 \mathrm{TeV}(n=2)$ & \\
Supernovae cooling rate [17]-[20] & $30 \mathrm{TeV}(n=2)$ & $2.5 \mathrm{TeV}(n=3)$ \\
Non-thermal production of KK modes [21] & $35 \mathrm{TeV}(n=2)$ & $3 \mathrm{TeV}(n=6)$ \\
Diffuse gamma-ray background [16]22] 23] & $110 \mathrm{TeV}(n=2)$ & $5 \mathrm{TeV}(n=3)$ \\
Thermal production of KK modes [23] & $167 \mathrm{TeV}(n=2)$ & $1.5 \mathrm{TeV}(n=5)$ \\
Neutron star core halo [24] & $500 \mathrm{TeV}(n=2)$ & $30 \mathrm{TeV}(n=3)$ \\
Time delay in photons from GRB's [25] & $620 \mathrm{TeV}(n=1)$ & \\
Neutron star surface temperature [24] & $700 \mathrm{TeV}(n=2)$ & $0.2 \mathrm{TeV}(n=6)$ \\
BH absence in neutrino cosmic rays [26] & & $1-1.4 \mathrm{TeV}(n \geq 5)$ \\
\hline
\end{tabular}

of $M_{*}$, then it may be shown that the electroweak symmetry breaking in the visible brane takes place at a scale [10]

$$
M_{E W}=e^{-k L} M_{*},
$$

where $k$ is the curvature scale associated with the negative cosmological constant that fills the 5-dimensional spacetime of the model. The effective Planck scale $M_{P}$ is now related to the fundamental one $M_{*}$ through the equation

$$
M_{P}^{2}=\frac{M_{*}^{3}}{k}\left(1-e^{-2 k L}\right) .
$$

In both scenaria, a low-scale gravitational theory can be realized in the context of the higher-dimensional model. As we will see, this will have important consequences for the creation and evaporation process of black holes in these theories. In these lectures, we will concentrate on the scenario with Large Extra Dimensions, however, many of the arguments and results that will be presented hold for the scenario with Warped Extra Dimensions, too, under the assumption that the AdS radius $1 / k$ is much larger than the horizon radius $r_{H}$ of the corresponding black holes.

\subsection{Creation of Black Holes}

A summary of the most important - experimental, astrophysical and cosmological limits on the fundamental energy scale $M_{*}$ is presented in Table 1 . From its entries 
one may see that, in general, the constraints become more relaxed as the number of additional spacelike dimensions increases. The most optimistic case is the one where the higher-dimensional Planck scale $M_{*}$ is very close to the TeV scale - this case is still viable, however, one needs to introduce at least 3 additional spacelike dimensions. In this version of the model the hierarchy between the gravitational and the electroweak scale almost disappears. What is more important, the scale of quantum gravity, where gravitational and SM interactions become of the same magnitude, approaches the energy scale where present-day and future experiment operate. As a result, if $M_{*}$ is of the order of a few TeV, then collider experiments with $E>M_{*}$ can probe the strong gravity regime and may witness the creation of heavy, extended objects!

The following question therefore arises naturally: can we then produce a black hole in a collider experiment on our brane? The idea was put forward in [27] very soon after the formulation of the two aforementioned models with extra dimensions. In there, it was argued that during a high-energy scattering process with $E>M_{*}$ and impact parameter $b$ between the colliding particles, the following two cases should be expected: (i) if $b>r_{H}(E)$, elastic and inelastic processes will take place, dominated by the exchange of gravitons, while (ii) if $b<r_{H}(E)$, a black hole will be formed according to the Thorne's Hoop Conjecture [28] and the colliding particles will disappear for ever behind the event horizon. In the above, $r_{H}(E)$ is the Schwarzschild radius that corresponds to the center-of-mass energy $E$ of the colliding particles.

Since gravity is higher-dimensional, every gravitational object, including the produced black hole, will be generically higher-dimensional. We thus expect the black hole to form on but also to extend off our brane. Under the assumption that the produced black hole has a horizon radius $r_{H}$ much smaller than the size of the extra dimensions $\mathscr{R}$ - a case that can be indeed realized as we will see in the next subsection, it may be assumed that it lives in a spacetime with $(4+n)$ non-compact dimensions. The simplest such black hole is the spherically-symmetric, neutral, higher-dimensional one described by the Schwarzschild-Tangherlini line-element [30]

$$
d s^{2}=-\left[1-\left(\frac{r_{H}}{r}\right)^{n+1}\right] d t^{2}+\left[1-\left(\frac{r_{H}}{r}\right)^{n+1}\right]^{-1} d r^{2}+r^{2} d \Omega_{2+n}^{2}
$$

where $d \Omega_{2+n}^{2}$ is the line-element of a $(2+n)$-dimensional unit sphere

$d \Omega_{2+n}^{2}=d \theta_{n+1}^{2}+\sin ^{2} \theta_{n+1}\left(d \theta_{n}^{2}+\sin ^{2} \theta_{n}\left(\ldots+\sin ^{2} \theta_{2}\left(d \theta_{1}^{2}+\sin ^{2} \theta_{1} d \varphi^{2}\right) \ldots\right)\right)$.

2 ... which says that "A black hole is formed when a mass M gets compacted into a region whose circumference in every direction is $\mathscr{C} \leq 2 \pi r_{H}(E)$ ". A higher-dimensional version of this conjecture was developed in [29] where the "circumference" was substituted by the "area" $\mathscr{V}_{D-3}$ of the $(D-3)$-dimensional "surface" that now needs to be $\mathscr{V}_{D-3} \leq G_{D} M$. 
Table 2 The values of the ratio $x_{\min }=E / M_{*}$, necessary for the creation of a black hole, as a function of $n$.

\begin{tabular}{cccccc}
\hline$n=2$ & $n=3$ & $n=4$ & $n=5$ & $n=6$ & $n=7$ \\
\hline$x_{\text {min }}=8.0$ & $x_{\text {min }}=9.5$ & $x_{\text {min }}=10.4$ & $x_{\text {min }}=10.9$ & $x_{\text {min }}=11.1$ & $x_{\text {min }}=11.2$ \\
\hline
\end{tabular}

By applying the Gauss law in $D=4+n$ dimensions, we find for the horizon radius the result [31]

$$
r_{H}=\frac{1}{M_{*}}\left(\frac{M_{B H}}{M_{*}}\right)^{\frac{1}{n+1}}\left(\frac{8 \Gamma\left(\frac{n+3}{2}\right)}{(n+2) \sqrt{\pi}^{(n+1)}}\right)^{1 /(n+1)}
$$

The above expression reveals the, by now, well-known result that, in an arbitrary number of dimensions, the horizon radius of the black hole has a power-law dependence on its mass $M_{B H}$ - the more familial linear dependence is restored if one sets $n=0$. More importantly, it is the fundamental Planck scale $M_{*}$ that appears in the denominator instead of the 4-dimensional one $M_{P}$, a feature that will play an important role on deciding whether black holes may be created at high-energy particle collisions.

Turning therefore to this question, the basic criterion for the creation of such a black hole is [32] that the Compton wavelength $\lambda_{C}=4 \pi / E$ of the colliding particle of energy $E / 2$ must lie within the corresponding Schwarzschild radius $r_{H}(E)$. By using the expression for the horizon radius $(10)$, the above is written as

$$
\frac{4 \pi}{E}<\frac{1}{M_{*}}\left(\frac{E}{M_{*}}\right)^{\frac{1}{n+1}}\left(\frac{8 \Gamma\left(\frac{n+3}{2}\right)}{(n+2) \sqrt{\pi}^{(n+1)}}\right)^{1 /(n+1)} .
$$

This inequality can be solved to give the ratio $x_{\min }=E / M_{*}$, necessary for the creation of the black hole. The results for $x_{\min }$ for various values of the number of extra dimensions $n$ are given in Table 2 From these, we conclude that the center-of-mass energy of the collision must be approximately one order of magnitude larger than the fundamental Planck scale $M_{*}$. Note that, if the factor $4 \pi$ is left out, as it was often done in earlier back-on-the-envelope calculations, the constraint on $E$ comes out to be much more relaxed, i.e. $E \geq M_{*}$. As the maximum center-of-mass energy that can be achieved at the Large Hadron Collider at CERN is $14 \mathrm{TeV}$, it seems that a window of approximately $5 \mathrm{TeV}$ remains at our disposal to witness a strong gravity effect such as the creation of a black hole.

Moving beyond the classical criterion (11) that allows for the formation of the black hole, two basic questions arise next: (i) what part of the available center-ofmass energy $E$ is absorbed inside the black hole, and (ii) how likely is the creation of a black hole at the first place. In order to answer these questions, we need to study the details of the high-energy particle collision in a strong gravitational background. A theory of Quantum Gravity could provide the answers, however, such a theory - 
Fig. 2 Two Aichelburg-Sexl shock waves propagating at opposite directions. The two shock waves collide at $u=v=0$ and, if a closed trapped surface or an apparent horizon is formed, a black hole has been created.

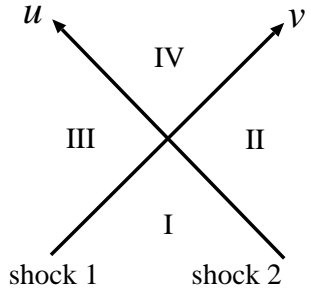

in a complete, consistent form - is still missing. Over the years the fast-moving, colliding particles have been modelled by gravitational waves, shock waves, and strings in the context of different theories such as General Relativity (with or without Quantum mechanics) [33, 34, 35, 36, 37, 38, 39], String Theory [40, 41, 42] and Topological Field Theory [43]. The most widely established method is the use of the concept of the Aichelburg-Sexl shock wave [44] that was developed more than twenty years ago in the context of a 4-dimensional gravitational theory. An Aichelburg-Sexl shock wave follows from a Schwarzschild line-element boosted along the $z$ axis, with a Lorentz factor $\gamma=1 / \sqrt{1-\beta^{2}}$. In the limit $\gamma \rightarrow \infty$, the boosted line-element becomes [37]

$$
d s^{2}=-d u d v+d x^{2}+d y^{2}+4 \mu \ln \left(x^{2}+y^{2}\right) \delta(u) d u^{2},
$$

with $u=t-z$ and $v=t+z$, and $\mu$ the particle's energy. The above line-element is everywhere flat apart from the point $u=0$ where a discontinuity arises. Therefore, it describes a shock wave located at this point and moving along the $+z$ axis at the speed of light.

We now assume that two Aichelburg-Sexl waves, with their centers at $u=0$ and $v=0$, are moving at opposite directions, one along the $+z$ axis and the other along the $-z$. Then, the two shock waves will collide at $u=v=0$, as we may see at Fig. 2 The points at regions I, II and III, that lie away from the moving trajectories and the collision point, are flat, however, the region IV, which forms after the collision is highly non-linear and curved 3 . If, at the union of the two shock waves, a closed trapped surface (i.e. a closed 2-dimensional spacelike surface on which the outgoing orthogonal null geodesics have positive convergence [45]) or an apparent horizon (that is, a closed trapped surface with exactly zero convergence) is formed, then a black hole has been created - since, according to the Cosmic Censorship Hypothesis, the apparent horizon either coincides with or lies inside the event horizon [45].

The creation therefore of a black hole is nothing but a Boundary Value problem. In $D=4$ dimensions and for a head-on collision $(b=0)$, this problem can be solved analytically. This task was performed by Penrose [46], more than 30 years ago, who found that an apparent horizon is indeed formed with an area equal to $32 \pi \mu^{2}$. This

\footnotetext{
${ }^{3}$ The task of finding the exact form of spacetime in region IV involves strong, and thus non-linear, gravitational calculations; until today no answer - analytical or numerical - has been given to this question.
} 
can put a lower bound on the area of the event horizon and thus on the black hole mass as follows:

$$
A_{H} \equiv 4 \pi r_{H}^{2} \geq 32 \pi \mu^{2} \Rightarrow M_{B H} \equiv \frac{r_{H}}{2} \geq \frac{1}{\sqrt{2}}(2 \mu) .
$$

From the above one may conclude that at least $71 \%$ of the initial energy $E=2 \mu$ of the collision is trapped inside the black hole. Alternatively, one may compute the amount of energy emitted in the form of gravitational waves during the violent collision. This was done in [37] where it was found that that amount was of the order of $16 \%$, which raised the percentage of energy absorbed by the black hole to $84 \%$ of the initial energy $E$. In more recent years, numerical analyses, where one [47] or both [48] of the colliding bodies were assumed to be described by a black hole, have found that the percentage of energy lost in the form of gravitational waves is in the area of $14 \%$, whish is in very good agreement with the results of [37].

In the brane-world scenario, the colliding particles need to enter the higherdimensional regime in order to create a black hole. In that case, every closed trapped surface will be a $(D-2)$ surface instead of a 2-dimensional. Nevertheless, the same procedure for investigating the creation of a black hole can be followed in this case, too. For a head-on collision, the corresponding boundary value problem can be again solved analytically leading to [49]

$$
M_{B H} \geq[0.71(\text { for } D=4)-0.58(\text { for } D=11)](2 \mu) .
$$

Therefore, as the dimensionality $D$ of spacetime increases, smaller and smaller black holes will be created. On the other hand, if the collision is not head-on, i.e. $b \neq 0$, numerical means have to be used to find the solution of the problem. This was done in [49, 50] leading respectively to the results

- $D=4: \quad b \leq b_{\max } \simeq 0.8 r_{H}$,

- $D=4+n: \quad b \leq b_{\max } \simeq 32^{-(n+2) /(n+1)} r_{H}$.

Thus, for a non-head-on collision, a black hole will be created if the impact parameter is smaller than a fraction of the event horizon radius. This fraction is 0.8 in $D=4$ and increases, reaching asymptotically unity, as $D$ becomes larger.

The impact parameter can offer us a measure of how likely the creation of a black hole is. For a high-energy collision with a non-zero impact parameter $b$, the production cross-section is found by using the geometric limit

$$
\sigma_{\text {production }} \simeq \pi b^{2} .
$$

According to the above, the cross-section for the production of black holes from two fast-moving particles is assumed to be given by the classical formula for the "target" area defined by the impact parameter. One might intuitively think that the formation of a black hole at such high energies would be governed by quantum, rather than classical, effects - in [51], the argument that the production cross-section would be suppressed by an exponential factor involving the Euclidean action of the 
Table 3 Black-Hole production cross-section as a function of the dimensionality of spacetime [57]

\begin{tabular}{ccccccccr}
\hline$D$ & 4 & 5 & 6 & 7 & 8 & 9 & 10 & 11 \\
\hline$\sigma_{\text {production }} /\left(\pi r_{H}^{2}\right)$ & 0.71 & 1.54 & 2.15 & 2.52 & 2.77 & 2.95 & 3.09 & 3.20 \\
\hline
\end{tabular}

system was put forward. However, in subsequent studies [52, 53, 54, 55, 56] it was shown that the creation of the black hole was a classically allowed process and not a quantum phenomenon; the main contribution to the production cross-section is therefore given by the classical expression (15) with the quantum corrections being indeed small as in [51].

Early studies used the approximate expression $\sigma_{\text {production }} \simeq \pi r_{H}^{2}$, but today a more precise expression is needed. As we just saw, even in the $D=4$ case, a black hole will not be created unless the impact parameter is smaller than $0.8 r_{H}$, a result that leads to the more accurate estimate for the production cross-section: $\sigma_{\text {production }} \simeq$ $0.64\left(\pi r_{H}^{2}\right)$. Nevertheless, we are not done: novel estimates for the production crosssection have emerged from the study of Ref. [57], where the search for the creation of a closed trapped surface was extended in the regime $(u=0, v>0)$ and $(u>0$, $v=0$ ), i.e. in the 'future' of the collision point. This extension gave a boost to the production cross-section, since in cases where it was previously concluded that no event horizon had been formed at the collision point, now such a surface was found when the extended regime was used instead. Therefore, the state-of-the-art values of the black hole production cross-section are the ones given in Table 3 [57. For example, the $D=4$ value of 0.64 , in units of $\pi r_{H}^{2}$, has increased to 0.71 , with similar or larger enhancements taking place for the other values of $D$, tod 4 .

Focusing, for a moment, on the geometrical instead of the numerical factor in the expression of the production cross-section, we may write

$$
\sigma_{\text {production }} \propto \pi r_{H}^{2} \sim \frac{1}{M_{*}^{2}}\left(\frac{E}{M_{*}}\right)^{2 /(n+1)} .
$$

The above expression gives the dependence of the production cross-section on the center-of-mass energy of the collision and reveals the enhancement of it with $E$, a dependence that is not seen in any other SM or Beyond the Standard Model proces.5. The above expression is valid for the production of a black hole out of two

\footnotetext{
${ }^{4}$ We should note here that the use of the generalized uncertainty principle has shown to lead to an increase in the minimum amount of energy needed for the creation of a black hole [58]. Similarly, the production cross-section comes out to be suppressed if the charge of the colliding particles exceeds a certain value [59], while the angular momentum of the black hole enhances $\sigma_{\text {production }}$ [60]. Finally, if one assumes the existence of a non-Gaussian point in General Relativity and thus a running gravitational coupling, the black-hole production cross-section is greatly suppressed in part of the parameter space [61].

${ }^{5}$ For a black hole produced in a 5-dimensional Anti de Sitter spacetime, the above result for the production cross-section holds if we assume that $r_{H} \ll 1 / k$; on the other hand, if $r_{H} \geq 1 / k$, then, the corresponding expression for the production cross-section is $\sigma_{\text {production }} \propto \ln ^{2} E[62]$.
} 
elementary, non-composite particles (i.e. partons). The final result for the production cross-section out of two accelerated composite particles, such as protons, follows by properly summing over all pairs of partons that carry enough energy to produce a black hole. This is finally given by [63, 64]

$$
\sigma_{\text {production }}^{p p \rightarrow B H}=\sum_{i j} \int_{\tau_{m}}^{1} d \tau \int_{\tau}^{1} \frac{d x}{x} f_{i}(x) f_{j}\left(\frac{\tau}{x}\right) \sigma_{\text {production }}^{i j \rightarrow B H},
$$

where $x$ is the parton-momentum fraction, $\tau=\sqrt{x_{i} x_{j}}$, and $f_{i}(x)$ are the so-called parton distribution functions (PDF's) that determine the fraction of the center-ofmass energy that is carried by the partons.

Summing over all possible pairs of partons gives another considerable boost to the production cross-section. One could naively think that by increasing without limit the available center-of-mass energy $E$, one could create extremely energetic pairs of partons each one of which would certainly create a black hole. However, the parton distribution functions $f_{i}(x)$ decrease rapidly with the center-of-mass en$\operatorname{ergy} E$, and with them the amount of energy that is passed to (and retained by) the partons. As a result, the production cross-section can not be indefinitely increased. Numerical calculations, that take into account the compositeness of the accelerated particles and the behaviour of PDF's, have derived some indicative values for $\sigma_{\text {production }}\left[63\right.$, 64]. For example, if we assume that $M_{*}=1 \mathrm{TeV}$ and $D=10$, then the production cross-section for a black hole with $M_{B H}=5 \mathrm{TeV}$ turns out to be $\sigma_{\text {production }} \sim 10^{5} \mathrm{fb}$, while for a black hole with $M_{B H}=10 \mathrm{TeV}$ it is found that $\sigma_{\text {production }} \sim 10 \mathrm{fb}$. For beyond the SM processes, the aforementioned values are quite significant - in the first case, the value of $\sigma_{\text {production }}$ amounts to one black hole created per second! Whether LHC will indeed prove to be a black-hole factory, it remains to be seen.

\subsection{Black-Hole Properties}

We now turn to the properties of the higher-dimensional black holes that may be produced during trans-planckian particle collisions [65, 66]. We will use as a prototype for our discussion the spherically-symmetric, neutral black hole described by the Schwarzschild-Tangherlini line-element (8). Let us start with the value of the horizon radius - how big (or, small) are actually these black holes? The value of $r_{H}$ as a function of the mass of the black hole is given in Eq. (10). In order to derive some realistic estimates, we assume again $M_{*}=1 \mathrm{TeV}$ and $M_{B H}=5 \mathrm{TeV}$, and calculate the value of the horizon as a function of the number of extra dimensions $n$. These values are presented in Table 4. From these we may easily conclude that, in the presence of extra dimensions, in order to create a black hole we only need to access subnuclear distances. To have a measure of comparison, let me note that, in $D=4$ with $M_{*}=M_{P} \simeq 10^{19} \mathrm{GeV}$, the same objective could only be achieved if the two colliding particles came within a distance of $10^{-35} \mathrm{fm}$ ! 
Table 4 Horizon radius and temperature of the Schwarzschild-Tangherlini black hole as a function of the number of extra dimensions, for $M_{*}=1 \mathrm{TeV}$ and $M_{B H}=5 \mathrm{TeV}$

\begin{tabular}{cccccccc}
\hline$n$ & 1 & 2 & 3 & 4 & 5 & 6 & 7 \\
\hline$r_{H}\left(10^{-4} \mathrm{fm}\right)$ & 4.06 & 2.63 & 2.22 & 2.07 & 2.00 & 1.99 & 1.99 \\
$T_{H}(\mathrm{GeV})$ & 77 & 179 & 282 & 379 & 470 & 553 & 629 \\
\hline
\end{tabular}

Drawing from our knowledge of their 4-dimensional analogues, we expect that these miniature black holes will go through the following stages during their lifetime [63]: (i) The balding phase: the initially highly asymmetric black hole will shed all quantum numbers and multipole moments apart from its mass $M$, electromagnetic charge $Q$, and angular momentum $J$ - during this phase, we expect some visible but mainly invisible energy emission. (ii) The spin-down phase: the black hole will start losing its angular momentum via the emission of Hawking radiation through mainly visible channels. (iii) The Schwarzschild phase: after its angular momentum the black hole will now start losing its mass through the emission again of Hawking radiation. (iv) The Planck phase: when the black hole $M_{B H}$ approaches $M_{*}$, it becomes a quantum object whose properties would follow only from a quantum theory of gravity - possible scenaria for this phase are the emission of a few energetic quanta leading to the complete evaporation of the black hole, or the formation of a stable "quantum" remnant.

The emission of Hawking radiation [67] is sourced by the non-vanishing temperature of the black hole. This is defined in terms of the black hole's surface gravity $k$ as follows

$$
T_{H}=\frac{k}{2 \pi}=\frac{1}{4 \pi} \frac{1}{\sqrt{\left|g_{t t} g_{r r}\right|}}\left(\frac{d\left|g_{t t}\right|}{d r}\right)_{r=r_{H}}=\frac{(n+1)}{4 \pi r_{H}} .
$$

By using again, as an indicative case, the values $M_{*}=1 \mathrm{TeV}$ and $M_{B H}=5 \mathrm{TeV}$, for the fundamental Planck scale and black-hole mass, as well as the values of the horizon radius $r_{H}$ given in Table 4, we may calculate the temperature of the black hole in terms of the number of extra dimensions. These are also given in Table 4 We observe that a higher-dimensional black hole, with mass in the range of values that would allow it to be produced at LHC, comes out to have in addition a temperature that would greatly facilitate its detection at present and future experiments unlike the large astrophysical black holes that are characterised by an extremely low temperature and the majority of primordial black holes that have an extremely high temperature. Finally, let us add that due to the emission of Hawking radiation, the lifetime of a black hole is finite. In the case of a higher-dimensional black hole, this quantity is given by [65]

$$
\tau_{(n+4)} \sim \frac{1}{M_{*}}\left(\frac{M_{B H}}{M_{*}}\right)^{\frac{(n+3)}{(n+1)}}>\tau_{(4)} .
$$


For the same values of $M_{*}$ and $M_{B H}$, the typical lifetime of the black hole comes out to be $\tau=(1.7-0.5) \times 10^{-26} \mathrm{sec}$ for $n=1-7$. In other words, the produced black hole will evaporate instantly after its creation, and it will do so right in front of our detectors.

That is why we need to study in the greatest possible detail the spectrum of the Hawking radiation emitted by the black hole as this will probably be the main observable effect associated with this gravitational object. Although a purely geometrical property, the temperature of a black hole leads to the emission of thermal radiation similar to that of a black body. The Hawking radiation [67] is therefore a classical phenomenon but with a quantum origin, since classically nothing is allowed to escape from within the black-hole horizon. The emission of radiation from a black hole, 4-dimensional and high-dimensional alike, can be realized through the creation of a virtual pair of particles just outside the horizon; when the antiparticle happens to fall inside the black hole, the particle can then propagate away from the black hole whose mass has decreased due to the negative amount of energy it received. The radiation spectrum is therefore a nearly black-body spectrum with energy emission rate given by an expression of the form [67]

$$
\frac{d E(\omega)}{d t}=\frac{|\mathscr{A}(\omega)|^{2} \omega}{\exp \left(\omega / T_{H}\right) \mp 1} \frac{d \omega}{(2 \pi)} .
$$

The quantity $|\mathscr{A}(\omega)|^{2}$ appearing in the numerator is the Absorption Probability (or, greybody factor). Its presence is due to the fact that a particle, propagating in the $(4+n)$-dimensional black-hole background, needs to escape the strong gravitational field, that the black hole creates, to reach the asymptotic observer. In order to see this, we may write the equation of motion of an arbitrary field in the aforementioned background in the form of a Schrödinger-like equation

$$
-\frac{d^{2} \Psi}{d r_{*}^{2}}+V\left(r_{*}, n, l, \omega, s, \ldots\right) \Psi=\omega^{2} \Psi,
$$

in terms of the so-called tortoise coordinate $d r_{*}=\left[1-\left(\frac{r_{H}}{r}\right)^{(n+1)}\right]^{-1} d r$. The gravitational barrier $V\left(r_{*}, n, l, \omega, s, \ldots\right)$ will reflect some particles back to the black hole while it will allow others to escape to infinity. The rate at which particles and therefore energy is "arriving" at the location of the asymptotic observer will thus be proportional to the transmission (or, absorption, as we will shortly see) probability and thus different from the one for the usual, flat-space blackbody radiation. However, the extra difficulty that the greybody factor introduces in the calculation of the radiation spectrum is compensated by the following fact: the barrier, and consequently the absorption probability, depends on a number of parameters that describe both particle properties ( $\operatorname{spin} s$, energy $\omega$, angular momentum numbers $l, m, \ldots$ ) and spacetime properties (number of extra dimensions $n$, angular momentum of black hole $a$, cosmological constant $\Lambda, \ldots$.). As a result, the Hawking radiation spectrum, when computed, is bound to be a vital source of information on the emitted particles and gravitational background. 
But how does the radiation spectrum follow? For this, we need to do a Quantum Field Theory analysis in curved spacetime. The first step is to define a basis for our fields: in the 4-dimensional case, we write [68, 69]

$$
u_{\omega l m}=\frac{N}{r} e^{-i \omega t} e^{i m \varphi} S_{\omega l m}(\theta) R_{\omega l m}(r),
$$

where $N$ is a normalization constant, $(l, m)$ the angular momentum numbers with $|m| \leq l$, and $S_{\omega l m}(\theta)$ the spherical harmonics. We also need to define the vacuum state of the theory. The one that describes perfectly the Hawking radiation emission process is the past Unruh vacuum $\left|U^{-}\right\rangle$: this state has no incoming radiation from past null infinity $\mathscr{J}^{-}$(i.e. far away from the $\mathrm{BH}$ at some asymptotic initial time) but modes can "come out" of the black hole.

The gravitational potential $V$ that appears in the equation of motion of the field propagating in the black-hole background has the form of a barrier: it is localised, and vanishes at both the horizon and infinity. At these two asymptotic regimes, Eq. (21) can then be easily solved, and the radial part of the field assumes the forms

$$
R_{\omega l m}^{u p}(r) \sim\left\{\begin{array}{ll}
e^{i \omega r_{*}}+A^{u p} e^{-i \omega r_{*}}, & r \rightarrow r_{H} \\
B^{u p} e^{i \omega r_{*}}, & r \rightarrow \infty
\end{array} .\right.
$$

The solution is, as expected, a superposition of free plane-waves, where the constants $A^{u p}$ and $B^{u p}$ can be viewed as the Reflection and Transmission coefficients.

The fluxes of particles $N$ and energy $E$ emitted by the black hole and measured by an observer at infinity are given by the vacuum expectation values of the radial component of the conserved current $J^{\mu}$ and the $(t r)$-component of the energymomentum tensor $T_{\mu \nu}$, respectively, evaluated at infinity [68]

$$
\frac{d^{2}}{r^{2} d t d \Omega}\left(\begin{array}{c}
N \\
E
\end{array}\right)=\left\langle U^{-}\left|\left(\begin{array}{c}
J^{r} \\
T^{t r}
\end{array}\right)\right| U^{-}\right\rangle_{\infty} .
$$

Using the asymptotic form (23) for the radial part of the field at infinity, and after some algebra, we find

$$
\frac{d^{2}}{d t d \omega}\left(\begin{array}{c}
N \\
E
\end{array}\right)=\frac{1}{2 \pi} \sum_{l} \frac{N_{l}\left|B^{u p}\right|^{2}}{\exp \left(\omega / T_{H}\right) \mp 1}\left(\begin{array}{c}
1 \\
\omega
\end{array}\right),
$$

where $N_{l}=2 l+1$ is the multiplicity of states that have the same value of the angular momentum number $l$, and the \pm 1 factor is a statistics factor for fermions and bosons, respectively.

We note that, in the numerator of the above expression, it is the transmission probability $\left|B^{u p}\right|^{2}$ that appears, as expected. However, one may define an alternative, but equivalent, basis, namely 


$$
R_{\omega l m}^{i n}(r) \sim\left\{\begin{array}{ll}
B^{i n} e^{-i \omega r_{*}}, & r \rightarrow r_{H} \\
e^{-i \omega r_{*}}+A^{i n} e^{i \omega r_{*}}, & r \rightarrow \infty
\end{array} .\right.
$$

This basis describes modes that originate not from the black hole but from the past null infinity. Now, $A^{i n}$ and $B^{i n}$ can be viewed as the Reflection and Absorption coefficients, respectively. As both sets of solutions satisfy the same radial equation, one may easily show that the following relations hold

$$
1-\left|A^{i n}\right|^{2}=\left|B^{i n}\right|^{2} \equiv\left|B^{u p}\right|^{2}=1-\left|A^{u p}\right|^{2} .
$$

From the above, we may easily conclude that the transmission probability $\left|B^{u p}\right|^{2}$ for the "up" modes originating from inside the black hole is equal to the absorption probability $\left|B^{i n}\right|^{2}$ for the "in" modes originating from past null infinity - we denote these two quantities collectively as $|\mathscr{A}(\omega)|^{2}$, and write

$$
\frac{d^{2}}{d t d \omega}\left(\begin{array}{l}
N \\
E
\end{array}\right)=\frac{1}{2 \pi} \sum_{l} \frac{N_{l}|\mathscr{A}(\omega)|^{2}}{\exp \left(\omega / T_{H}\right) \mp 1}\left(\begin{array}{c}
1 \\
\omega
\end{array}\right) .
$$

In the case of a rotating (Kerr) black hole, we may compute three rates: the emission rates of particles $N$ and energy $E$ and the rate of loss of the angular momentum $J$ of the black hole. These are given by the expressions

$$
\frac{d^{2}}{r^{2} d t d \Omega}\left(\begin{array}{c}
N \\
E \\
J
\end{array}\right)=\left\langle U^{-}\left|\left(\begin{array}{c}
J^{r} \\
T^{t r} \\
T_{\varphi}^{r}
\end{array}\right)\right| U^{-}\right\rangle_{\infty} .
$$

The asymptotic solutions for the radial part of the field for either the "up" modes or the "in" modes propagating in a Kerr black-hole background are now given by

$$
R_{\omega l m}^{u p}(r) \sim \begin{cases}e^{i \tilde{\omega} r_{*}}+A^{u p} e^{-i \tilde{\omega} r_{*}}, & r \rightarrow r_{H} \\ B^{u p} e^{i \omega r_{*}}, & r \rightarrow \infty\end{cases}
$$

and

$$
R_{\omega l m}^{i n}(r) \sim\left\{\begin{array}{ll}
B^{i n} e^{-i \tilde{\omega} r_{*}}, & r \rightarrow r_{H} \\
e^{-i \omega r_{*}}+A^{i n} e^{i \omega r_{*}}, & r \rightarrow \infty
\end{array} .\right.
$$

In the above, the parameter $\tilde{\omega}$ is defined as

$$
\tilde{\omega} \equiv \omega-m \Omega_{H}=\omega-m \frac{a}{r_{H}^{2}+a^{2}},
$$

where $\Omega_{H}$ is the angular velocity of the rotating black hole, and $a$ the angular momentum parameter to be defined later. By using as a basis the "up" modes, that, as we saw, describe more accurately the Hawking radiation emission process, we find the expressions 


$$
\frac{d^{2}}{d t d \omega}\left(\begin{array}{c}
N \\
E \\
J
\end{array}\right)=\frac{1}{2 \pi} \sum_{l, m} \frac{\omega}{\tilde{\omega}} \frac{\left|B^{u p}\right|^{2}}{\exp \left(\tilde{\omega} / T_{H}\right) \mp 1}\left(\begin{array}{c}
1 \\
\omega \\
m
\end{array}\right) .
$$

As in the non-rotating case, we also find that the following relations hold between the coefficients of the asymptotic solutions (30)-(31) for the two sets of modes

$$
\frac{\omega}{\tilde{\omega}}\left|B^{u p}\right|^{2}=1-\left|A^{u p}\right|^{2} \equiv 1-\left|A^{i n}\right|^{2}=\frac{\tilde{\omega}}{\omega}\left|B^{i n}\right|^{2},
$$

leading to the final, simpler formula for the three rates

$$
\frac{d^{2}}{d t d \omega}\left(\begin{array}{c}
N \\
E \\
J
\end{array}\right)=\frac{1}{2 \pi} \sum_{l, m} \frac{|\mathscr{A}(\omega)|^{2}}{\exp \left(\tilde{\omega} / T_{H}\right) \mp 1}\left(\begin{array}{c}
1 \\
\omega \\
m
\end{array}\right),
$$

where now $|\mathscr{A}(\omega)|^{2} \equiv=1-\left|A^{u p}\right|^{2} \equiv 1-\left|A^{i n}\right|^{2}$. Let us also note that if $\tilde{\omega}=\omega-$ $m \Omega_{H}<0$, then from Eq. (34) the reflection probabilities $\left|A^{u p}\right|^{2}$ and $\left|A^{i n}\right|^{2}$ can be larger than unity - this happens only for modes with $m>0$ and signals the effect of superradiance [70], where the incident wave "steals" energy from the rotating black hole and escapes with an amplitude larger than the original one.

Let us now introduce a number of additional, spacelike dimensions in our theory. Surprisingly, not much changes in the functional form of the above formulae. The emission rates for a higher-dimensional, rotating black hole will still be given by expressions of the form $[71,72,60,73,74,75,76,77,78,79]$

$$
\frac{d^{2}}{d t d \omega}\left(\begin{array}{c}
N \\
E \\
J
\end{array}\right)=\frac{1}{2 \pi} \sum_{l, m, j \ldots} \frac{|\mathscr{A}(\omega)|^{2}}{\exp \left(\tilde{\omega} / T_{H}\right) \mp 1}\left(\begin{array}{c}
1 \\
\omega \\
m
\end{array}\right) .
$$

Where does the difference from the 4-dimensional case lie? To start with, the temperature of the black hole will acquire an $n$-dependence. In addition, the equation of motion of a given field is going to depend on the specific background; therefore, the greybody factor $|\mathscr{A}(\omega)|^{2}$, that follows by solving the corresponding equation of motion is going to change, too. Also, the symmetry and structure of the higherdimensional spacetime may introduce additional quantum numbers and/or change the multiplicities of states that carry the same sets of quantum numbers.

Another important factor is whether we are considering emission of particles on the brane or in the bulk. Unlike a purely 4-dimensional black hole, a higherdimensional one can emit particles either in the "brane channel" or in the "bulk channel". The species of particles that can be emitted in the bulk are particles that are allowed by the model to propagate in the higher-dimensional spacetime, namely gravitons but also scalar fields that carry no quantum numbers under the SM gauge group. These bulk modes "see" the full $(4+n)$-dimensional gravitational background and they are invisible to us, therefore, any energy emitted in the bulk will be interpreted as a missing energy signal for a brane observer. On the other hand, the black hole can emit a variety of particles in the "brane channel", namely 
fermions, gauge bosons and Higgs-like scalars. These brane-localised modes "see" only the projected-on-the-brane 4-dimensional gravitational background and they are directly visible to a brane observer; as a result, they are the most interesting emission channel to study from the phenomenological point of view.

\section{Second Lecture: Hawking Radiation Spectra and Observable Signatures}

Having discussed the properties of the miniature black holes that may be created during a high-energy particle collision in the context of a low-scale higherdimensional gravitational theory, we now proceed to discuss in more detail the spectra of the Hawking radiation emitted by these black holes and the information on particle and spacetime properties that we may deduce from them.

\subsection{The Schwarzschild Phase on the Brane}

As we mentioned in the previous section, a black hole emits Hawking radiation during the two intermediate phases of its life, namely the spin-down and the Schwarzschild phase. We will start from the latter one, which although follows the spin-down phase, was the first one to be studied due to the simpler form of the line-element that describes the gravitational background around it. This is given by the Schwarzschild-Tangherlini solution (8) and describes, as we have seen, a spherically-symmetric, neutral black hole that has lost all of its angular momentum. For the purpose of studying the emission of Hawking radiation directly on the brane, we will be interested in the brane-localised modes that "see" only the

projected-on-the-brane background. In order to derive the latter, we fix the values of all the additional $\theta_{i}$ coordinates, with $i=2, \ldots, n+1$, introduced to describe the additional spacelike dimensions, to $\frac{\pi}{2}$. Then, the resulting brane background assumes the form

$$
d s_{4}^{2}=-\left[1-\left(\frac{r_{H}}{r}\right)^{n+1}\right] d t^{2}+\left[1-\left(\frac{r_{H}}{r}\right)^{n+1}\right]^{-1} d r^{2}+r^{2} d \Omega_{2}^{2} .
$$

The above line-element describes a 4-dimensional black-hole background on the brane which, although resembles a Schwarzschild background, it is distinctly different as it carries a non-trivial $n$-dependence. The horizon radius is still given by Eq. (10) and its temperature by Eq. (18) - note, that both the horizon radius and black-hole temperature follow from geometrical arguments involving only the $g_{t t}$ and $g_{r r}$ metric components, and these are not affected by the projection of the $(4+n)$-dimensional line-element onto the brane. 
However, the different form of the gravitational background is bound to change the equation of motion of the relevant species of particles, and thus the value of the greybody factor $|\mathscr{A}(\omega)|^{2}$. In order to study in a combined way the behaviour of fields with spin $s=0,1 / 2$ and 1 , a "master" equation of motion with $s$ appearing as a parameter was derived in $[71,72,66]$. For this, we used a factorized ansatz for the wavefuction of the field of the form

$$
\Psi_{s}=e^{-i \omega t} e^{i m \varphi} \Delta^{-s} R_{S}(r) S_{s l}^{m}(\theta),
$$

and employed the Newman-Penrose method [80, 81], that combines multi-component fields with curved gravitational backgrounds. Then, two decoupled equations, one for the radial function $R_{S}(r)$ and one for the spin-weighted spherical harmonics [82] $S_{s l}^{m}(\theta)$, were derived having the form

$$
\Delta^{s} \frac{d}{d r}\left(\Delta^{1-s} \frac{d R_{s}}{d r}\right)+\left[\frac{\omega^{2} r^{2}}{h}+2 i \omega s r-\frac{i s \omega r^{2} h^{\prime}}{h}-\lambda_{s l}\right] R_{s}(r)=0,
$$

and

$$
\frac{1}{\sin \theta} \frac{d}{d \theta}\left(\sin \theta \frac{d S_{s l}^{m}}{d \theta}\right)+\left[-\frac{2 m s \cot \theta}{\sin \theta}-\frac{m^{2}}{\sin ^{2} \theta}+s-s^{2} \cot ^{2} \theta+\lambda_{s l}\right] S_{s l}^{m}(\theta)=0,
$$

respectively. In the above, we have defined the function $\Delta \equiv r^{2} h \equiv r^{2}\left[1-\left(\frac{r_{H}}{r}\right)^{n+1}\right]$, while $\lambda_{s l}=l(l+1)-s(s-1)$ is the eigenvalue of the spin-weighted spherical harmonics. The above equations resemble the ones derived by Teukolsky [83] in the background of a purely 4-dimensional black-hole background and differ only in the expressions of the functions $h(r)$ and $\Delta(r)$.

The radial equation, from where the value of the greybody factor will follow, may be solved either analytically or numerically. If the analytic approach is chosen [71, 72], an approximation method must be followed according to which: (i) we solve the equation of motion in the Near-Horizon (NR) regime $\left(r \simeq r_{H}\right)$ where it takes the form of a hypergeometric equation, (ii) then, we solve the equation of motion in the Far-Field (FF) regime $\left(r \gg r_{H}\right)$ where it takes the form of a confluent hypergeometric equation, and (iii) finally, we match the two asymptotic solutions in an intermediate zone to guarantee the existence of a smooth solution over the whole radial regime. Once the solution for the radial function $R_{s}(r)$ is found, we compute the Absorption Probability (we use the "in" modes as a basis) through the formula

$$
|\mathscr{A}(\omega)|^{2} \equiv 1-|\mathscr{R}(\omega)|^{2} \equiv \frac{\mathscr{F}_{\text {horizon }}}{\mathscr{F}_{\text {infinity }}}
$$

where $\mathscr{R}(\omega)$ is the Reflection coefficient and $\mathscr{F}$ the flux of energy towards the black hole.

Whereas the Absorption Probability is a dimensionless quantity varying between 0 and 1 (in the non-rotating case), a dimensionful quantity may be constructed out of it, namely the absorption cross-section, that is measured in units of the horizon 

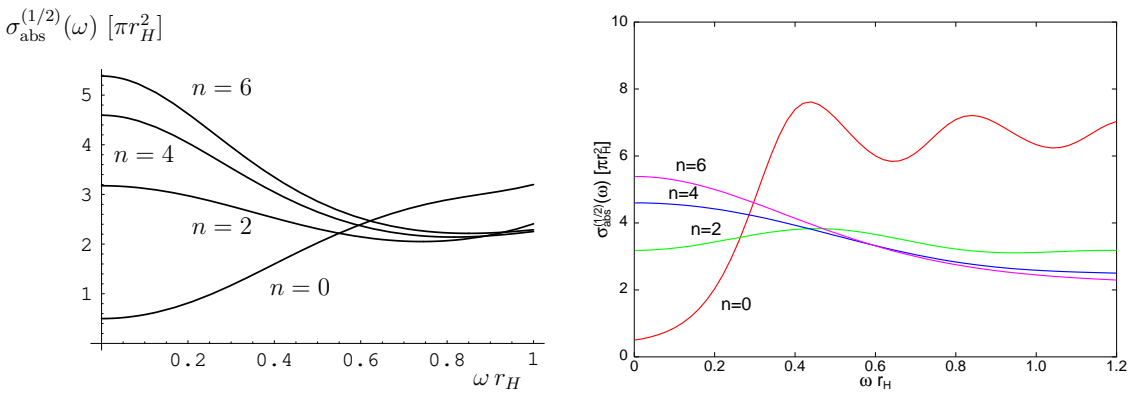

Fig. 3 Absorption cross-section for brane-localised fermions evaluated analytically (left plot), and numerically (right plot).

area $\left(\pi r_{H}^{2}\right)$ and is defined as [84]

$$
\sigma_{\mathrm{abs}}(\omega)=\sum_{l} \frac{\pi r_{H}^{2}}{\left(\omega r_{H}\right)^{2}}(2 l+1)|\mathscr{A}(\omega)|^{2}
$$

By following the approximate method, described above, to solve the radial equation, one may compute the absorption probability and from that the absorption crosssection. As an indicative case, in Fig. 3 (a) we present the result for $\sigma_{\mathrm{abs}}(\omega)$ for the case of fermions propagating in the projected-on-the-brane black-hole background. As we observe, the horizontal axis does not extend to large values of the energy parameter $\omega r_{H}$; the reason for this is that, during the matching of the two asymptotic solutions, the assumption was made that $\omega r_{H} \ll 1$, that inevitably restricts the validity of the analytic result to small values of the energy. Therefore, the behaviour of $\sigma_{\text {abs }}(\omega)$, or $\mathscr{A}(\omega)$, for arbitrary values of the energy can only be derived if numerical techniques are employed [85] for the solution of the radial equation. Then, the plot appearing in Fig. 3 b) can be constructed. The qualitative agreement between the two plots is obvious and one can see that the low-energy behaviour of $\sigma_{\mathrm{abs}}(\omega)$ is accurately reproduced by the analytic result. However, as $\omega r_{H}$ increases, deviations start appearing. To complete the picture, in Figs. 4 4 a,b) we present the behaviour of the absorption cross-section for scalars and gauge bosons [85], respectively. What is important in the behaviour of $\sigma_{\mathrm{abs}}(\omega)$ is that (a) it behaves differently for each species of fields, and (b) has a rather strong dependence on the number of spacelike dimensions that exist transversely to the brane.

When the (numerically) computed absorption probability and the temperature of the black hole are substituted in the formula for the energy emission rate, we obtain the radiation spectrum [85] that, for the indicative case of fermions, is depicted in Fig. 5. The different curves on the plot stand for the differential energy emission rates per unit time and unit frequency for the cases with $n=0,1,2,4$ and 6 (from bottom to top). We may easily observe that the energy emission rate is greatly enhanced by the number of extra spacelike dimensions, a result that holds also for scalars and gauge bosons. In order to derive the total emissivity, i.e. the energy emitted over the whole frequency regime per unit time, we integrate over $\omega r_{H}$. The results for 

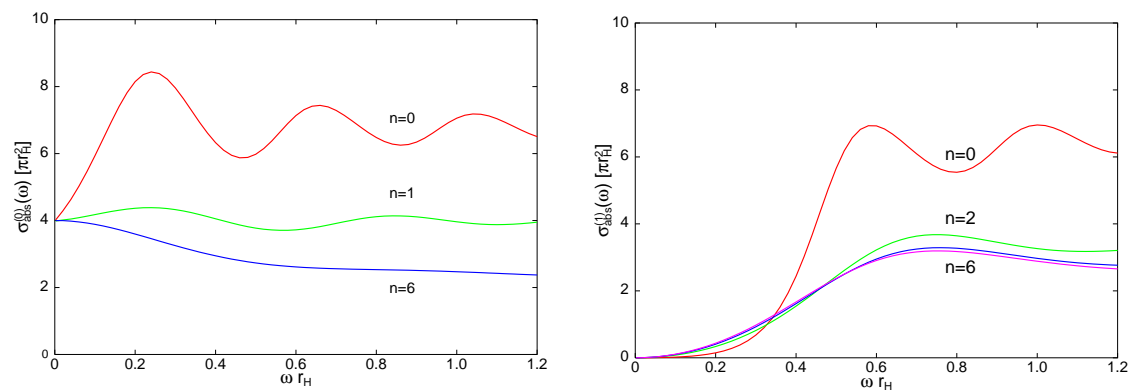

Fig. 4 Absorption cross-section for brane-localised scalars (left plot) and gauge bosons (right plot).

all species of brane-localised fields are presented in the first three rows of Table 5 . From there, we may see that the total emissivity for the SM fields is enhanced up to three or four orders of magnitude with the number of extra dimensions.

We finish this subsection with an interesting observation that applies for the relative emissivities of brane-localised fields. We have already seen that the absorption cross-section, and consequently the absorption probability, has a strong dependence on the spin of the propagating field. One thus expects that different species of particles will have different emission rates. Indeed, this may be seen by putting the emission curves of scalars, fermions and gauge bosons on the same graph. For a purely 4-dimensional black hole [86], this is shown in Fig. 6 a). According to this, the dominant type of particles emitted by a black hole in 4 dimensions is scalars; then come the fermions and finally the gauge bosons. Figure 6 b) shows the same emission curves but in the case of a 10-dimensional black hole. Here, the gauge bosons are the particles preferably emitted by the black hole, then come the scalars and lastly the fermions. Therefore, the number of extra dimensions determines not only the amount of energy emitted per unit time by the black hole but also the type of the emitted particles.

Fig. 5 Hawking radiation energy emission rates for brane-localised fermions, for $n=0,1,2,4$ and 6 (from bottom to top).

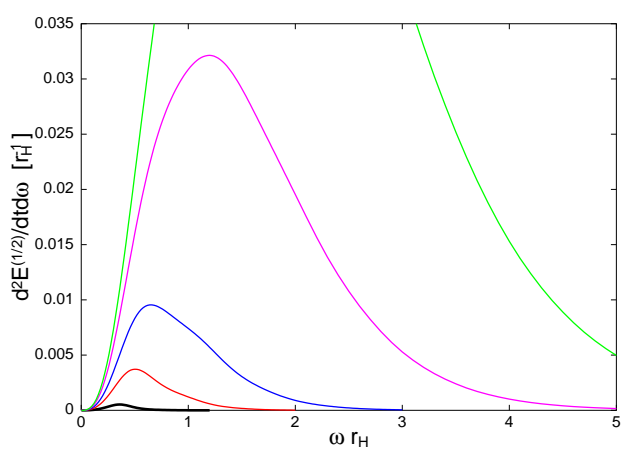


Table 5 Total emissivities for brane-localised scalars, fermions and gauge bosons [85] and bulk gravitons 98

\begin{tabular}{ccccccccc}
\hline$n$ & 0 & 1 & 2 & 3 & 4 & 5 & 6 & 7 \\
\hline Scalars & 1.0 & 8.94 & 36.0 & 99.8 & 222 & 429 & 749 & 1220 \\
Fermions & 1.0 & 14.2 & 59.5 & 162 & 352 & 664 & 1140 & 1830 \\
G. Bosons & 1.0 & 27.1 & 144 & 441 & 1020 & 2000 & 3530 & 5740 \\
Gravitons & 1.0 & 103 & 1036 & 5121 & $2 \times 10^{4}$ & $7 \times 10^{4}$ & $2.5 \times 10^{5}$ & $8 \times 10^{5}$ \\
\hline
\end{tabular}
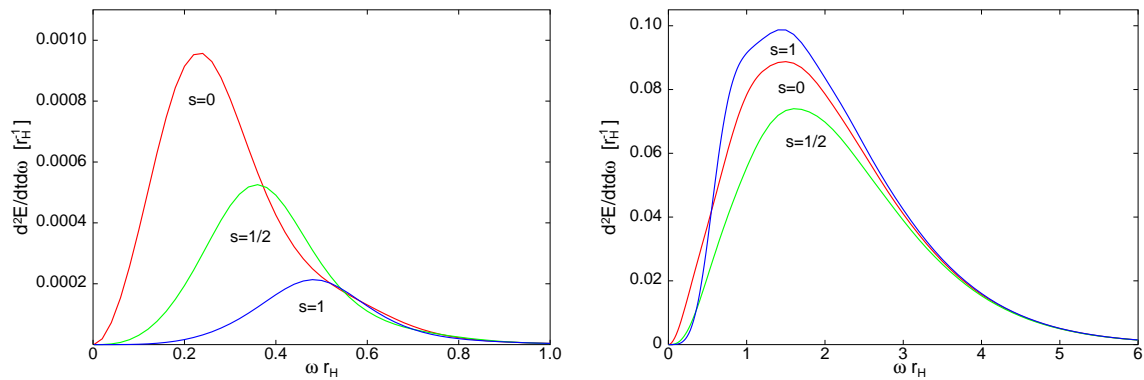

Fig. 6 Relative emissivities for brane-localised fields for $n=0$ (left plot) and $n=6$ (right plot).

\subsection{The Spin-down Phase on the Brane}

We now turn to the phase in the life of the black hole that precedes the Schwarzschild one. This is the spin-down phase during which the black hole has a non-vanishing angular momentum - this is the most generic situation for a black hole created by a non-head-on particle collision. Assuming that the produced black hole has an angular-momentum component only along an axis in our 3-dimensional space, the line-element that describes the gravitational background around such a higherdimensional black hole is given by the Myers-Perry solution [31]

$$
\begin{aligned}
d s^{2} & =\left(1-\frac{\mu}{\Sigma r^{n-1}}\right) d t^{2}+\frac{2 a \mu \sin ^{2} \theta}{\sum r^{n-1}} d t d \varphi-\frac{\Sigma}{\Delta} d r^{2} \\
& -\Sigma d \theta^{2}-\left(r^{2}+a^{2}+\frac{a^{2} \mu \sin ^{2} \theta}{\Sigma r^{n-1}}\right) \sin ^{2} \theta d \varphi^{2}-r^{2} \cos ^{2} \theta d \Omega_{n}^{2},
\end{aligned}
$$

where

$$
\Delta=r^{2}+a^{2}-\frac{\mu}{r^{n-1}}, \quad \Sigma=r^{2}+a^{2} \cos ^{2} \theta .
$$

The parameters $\mu$ and $a$ that appear in the metric tensor are associated to the black hole mass and angular momentum, respectively, through the relations

$$
M_{B H}=\frac{(n+2) A_{2+n}}{16 \pi G} \mu \quad \text { and } \quad J=\frac{2}{n+2} a M_{B H}
$$


where $A_{2+n}$ is the area of a $(2+n)$-dimensional unit sphere. The horizon radius is found by setting $\Delta\left(r_{H}\right)=0$ and is found to be: $r_{H}^{n+1}=\mu /\left(1+a_{*}^{2}\right)$, where we have defined the quantity $a_{*} \equiv a / r_{H}$. Finally, the temperature and rotation velocity of this black hole are given by

$$
T_{H}=\frac{(n+1)+(n-1) a_{*}^{2}}{4 \pi\left(1+a_{*}^{2}\right) r_{H}}, \quad \Omega_{H}=\frac{a}{\left(r_{H}^{2}+a^{2}\right)} .
$$

Since we are still interested in the emission of brane-localised modes by the black hole, we should first determine the line-element on the brane. As in the case of the Schwarzschild phase, this will follow by fixing the values of the "extra" angular coordinates. This results in the disappearance of the $d \Omega_{n}^{2}$ part of the metric leaving the remaining unaltered. Then, by employing again the Newman-Penrose method, we compute the two - decoupled again - master equations, one for the radial part of the field and one for the angular part, namely [66, 77]

$$
\Delta^{-s} \frac{d}{d r}\left(\Delta^{s+1} \frac{d R_{s}}{d r}\right)+\left[\frac{K^{2}-i K s \Delta^{\prime}}{\Delta}+4 i s \omega r+s\left(\Delta^{\prime \prime}-2\right) \delta_{s,|s|}-\Lambda_{s j}^{m}\right] R_{s}=0
$$

and

$$
\begin{aligned}
\frac{1}{\sin \theta} \frac{d}{d \theta}\left(\sin \theta \frac{d S_{s j}^{m}}{d \theta}\right)+[ & -\frac{2 m s \cot \theta}{\sin \theta}-\frac{m^{2}}{\sin ^{2} \theta}+a^{2} \omega^{2} \cos ^{2} \theta \\
& \left.\quad-2 a \omega s \cos \theta+s-s^{2} \cot ^{2} \theta+\lambda_{s j}\right] S_{s j}^{m}(\theta)=0 .
\end{aligned}
$$

In the above, $S_{s j}^{m}(\theta)$ are the spin-weighted spheroidal harmonics [87], and we have used the definitions

$$
K=\left(r^{2}+a^{2}\right) \omega-a m, \quad \Lambda_{s j}^{m}=\lambda_{s j}+a^{2} \omega^{2}-2 a m \omega .
$$

The angular eigenvalue $\lambda_{s j}$ does not exist in closed form but it may be computed either analytically, through a power series expansion in terms of the parameter $a \omega$ of the form [88, 89, 90]

$$
\lambda_{s j}=-s(s+1)+\sum_{k} f_{k}^{j m s}(a \omega)^{k},
$$

or numerically [74, 75, 77, 79].

The differential emission rates for the brane-localised modes during the spindown phase will be given by the 4-dimensional formula (35) but with the greybody factor computed from the brane equation of motion (47) and the temperature given by Eq. (46). Despite the complexity of the gravitational background, the absorption probability $|\mathscr{A}(\omega)|^{2}$ can be again found analytically in the low-energy and lowangular-momentum regime. For example, in the case of scalar fields, the dependence of $|\mathscr{A}(\omega)|^{2}$ on the angular momentum parameter $a$ and number of extra dimensions $n$ is given in Fig. 7 [91]. Each curve in the two plots is actually consisting of two 

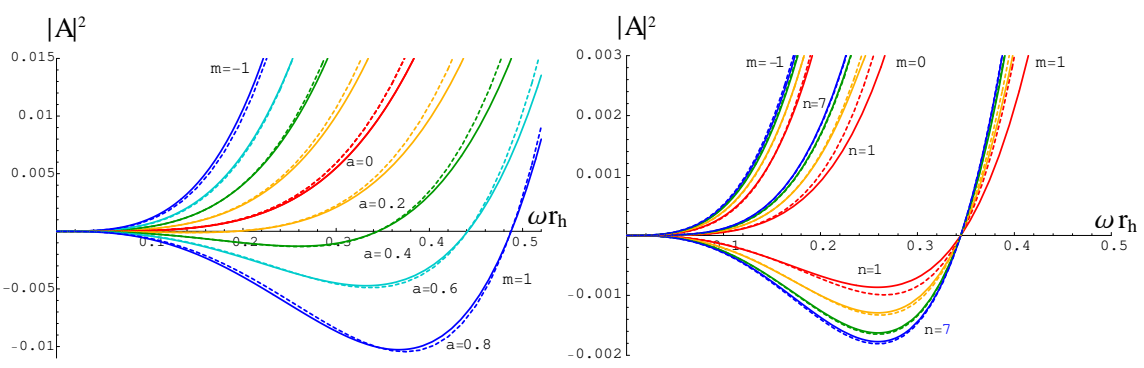

Fig. 7 Absorption probabilities for brane-localised scalar fields as a function of the angularmomentum parameter $a$ (left plot) and number of extra dimensions $n$ (right plot).

lines: a solid one, representing our analytic result, and a dotted one, representing the numerical result; it is clear that in the low- $\omega$ regime, the agreement between the two sets of results is indeed remarkable. A similar agreement is observed for the cases of fermions and gauge bosons [92].

However, for the complete spectrum, we have to retort again to numerical analysis [74, 75, 76, 77, 78, 79]. In Fig. 8, we present the energy emission rates, for the indicative cases of brane-localised scalars and gauge bosons, in terms again of the angular-momentum parameter and number of extra dimensions. It is clear that an increase in any of these two parameters results in the significant enhancement of the energy emission rate. In Table 6 we have put together the factors by which the energy emission rates are enhanced, in terms of $a$ and $n$, for brane-localised scalars [74, 75], gauge bosons [77] and fermions [79]. When the angular momentum parameter increases from 0 to 1 , the energy emission rates, for an 8-dimensional black hole, increases by a factor from 3 to 6 , whereas, for a black hole with a fixed angular momentum parameter $a_{*}=1$, the enhancement factor is of the order of 50-100 when $n$ increases from 1 to 7 . If we finally compare the relative emissivities of different species of fields, then, once again, it is the gauge bosons that a higher-dimensional rotating black hole prefers to emit on the brane.
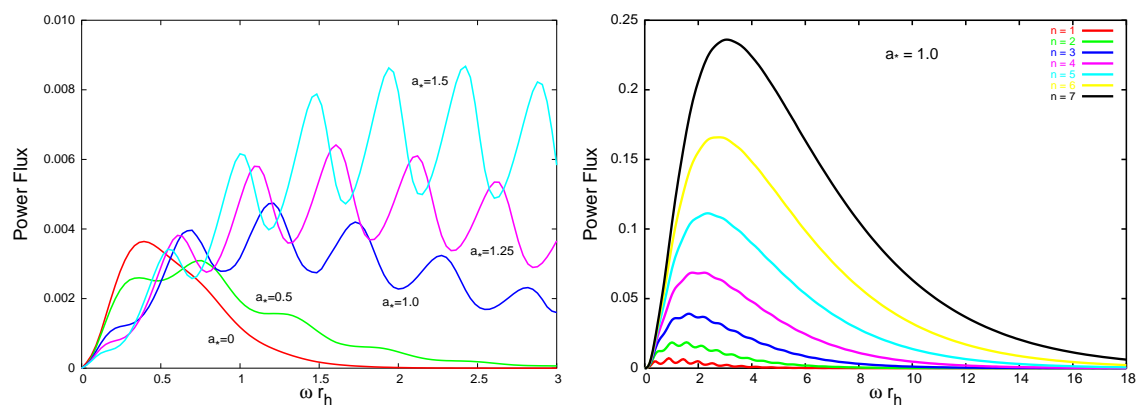

Fig. 8 Energy emission rates for brane-localised scalar fields in terms of the angular parameter (left plot) and gauge bosons in terms of the number of extra dimensions (right plot). 
Table 6 Enhancement factors for the energy emission rates in terms of the angular momentum parameter and number of extra dimensions

\begin{tabular}{c|ccc|ccc}
\hline & $(n=4)$ & $a_{*}=0$ & $a_{*}=1.0$ & $\left(a_{*}=1\right)$ & $n=1$ & $n=7$ \\
\hline Scalars & & 1 & $\geq 3$ & & 1 & $\geq 100$ \\
Fermions & 1 & 6 & 1 & 99 \\
G. Bosons & & 1 & $\geq 5$ & 1 & $\geq 50$ \\
\hline
\end{tabular}

Let us finally comment on a particular feature that the radiation spectra from the spin-down phase in the life of the black hole have. Unlike the line-element that describes the background around the black hole during its spherically-symmetric Schwarzschild phase, the one for the spin-down phase possesses a preferred axis in space, that is the rotation axis of the black hole. As a result, the radiation spectra of all emitted particles have a non-trivial angular dependence. As an indicative case, in Fig. 9. we present the energy emission rates for scalars, fermions and gauge bosons, from a 6-dimensional, rotating black hole with $a_{*}=1$, as a function of the energy parameter $\omega r_{H}$ and the $\cos (\theta)$ of the angle measured from the rotation axis of the black hole. In all spectra, we observe that most of the energy is emitted along the equatorial plane $(\theta=\pi / 2)$ as a result of the centrifugal force that is exerted on all species of fields. In the special cases of fermions and gauge bosons, i.e. of particles with non-vanishing spin, there is another effect, that of the spin-rotation coupling, that causes an additional angular dependence in their spectra, and aligns the emission along the rotation axis of the black hole - the effect is more dominant for gauge bosons than for fermions, and it dies out as the energy of the emitted particles increases. The angular spectra depicted in Fig. 9 follow after solving numerically the angular master equation (48) for the value of the spin-weighted spheroidal harmon$\operatorname{ics} S_{s j}^{m}(\theta)$ and calculating the differential emission rate

$$
\frac{d^{3} E}{d(\cos \theta) d t d \omega}=\frac{1}{4 \pi} \sum_{j=1}^{\infty} \sum_{m=-j}^{j} \frac{\omega|\mathscr{A}(\omega)|^{2}}{\exp \left(\tilde{\omega} / T_{H}\right)-1}\left[\left(S_{|s| j}^{m}\right)^{2}+\left(S_{-|s| j}^{m}\right)^{2}\right],
$$

per unit time, frequency and solid angle for each species of particles [75, 77, 79].

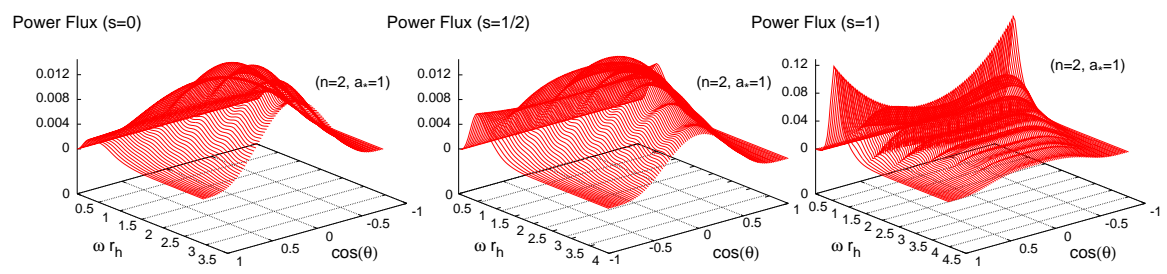

Fig. 9 Angular distribution of the energy emission spectra for scalars (left plot), fermions (central plot) and gauge bosons (right plot) for a 6-dimensional black hole with $a_{*}=1$. 
Fig. 10 Energy emission rates for bulk scalar fields, as a function of the number of additional spacelike dimensions, for the Schwarzschild phase.

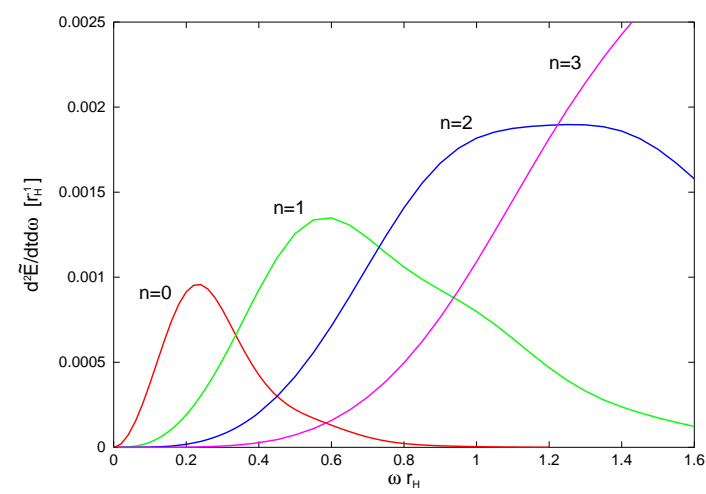

\subsection{Emission in the Bulk}

In the case that higher-dimensional mini black holes can indeed be created during particle collisions, their detection becomes more likely if a significant part of the black hole energy is channeled, through Hawking radiation emission, into brane fields. Therefore, although the bulk emission will be interpreted as a missing energy signal by a brane observer, we need to know the fraction of the total energy which is lost along this channel. We thus need to study the emission by the black hole of the species of particles that are allowed to propagate in the bulk, that is gravitons and possibly scalar fields. The latter are the easier to study as their equation of motion in the higher-dimensional spacetime can be easily found, by generalizing its 4-dimensional expression, to be

$$
\frac{1}{\sqrt{-G}} \partial_{M}\left[\sqrt{-G} G^{M N} \partial_{N} \Phi\right]=0,
$$

where the capital indices take values in the range $(0,1,2,3, \ldots, 4+n)$ and $G_{M N}$ is the metric tensor of the higher-dimensional spacetime.

We will study first the Schwarzschild phase, for which more results are available in the literature. In that case, the gravitational background that we need to consider is the higher-dimensional Schwarzschild-Tangherlini one (8). By assuming again a factorised ansatz for its wavefunction [85]

$$
\Phi\left(t, r, \theta_{i}, \varphi\right)=e^{-i \omega t} R_{\omega l}(r) \tilde{Y}(\Omega),
$$

where $\tilde{Y}(\Omega)$ is the higher-dimensional spherical harmonics [93], the equation of motion of the scalar field can reduce to a system of decoupled, radial and angular, equations. From the radial one, we find the absorption probability $|\mathscr{A}(\omega)|^{2}$ for a bulk scalar field, and finally the radiation spectrum [85]. This is given in Fig. 10 in terms of the number of the additional spacelike dimensions $n$. As in the case of brane emission, the energy emission rate for bulk scalar fields is greatly enhanced as $n$ increases. 
Table 7 Bulk-to-Brane Relative Emissivities Ratio for scalar fields in terms of $n$

\begin{tabular}{c|cccccccc}
\hline & $n=0$ & $n=1$ & $n=2$ & $n=3$ & $n=4$ & $n=5$ & $n=6$ & $n=7$ \\
\hline Bulk/Brane & 1.0 & 0.40 & 0.24 & 0.22 & 0.24 & 0.33 & 0.52 & 0.93 \\
\hline
\end{tabular}

Therefore, the question "which scalar channel, bulk or brane, is the most dominant one?" naturally arises. If the black hole has the choice to emit scalar fields both on the brane and in the bulk, which channel is the most effective? In order to answer this question, we need to compute the Bulk-to-Brane Relative Emissivity. This follows by integrating the corresponding brane and bulk spectra for scalar fields over the energy parameter $\omega r_{H}$, and computing their ratio. Then, we obtain the values for the Bulk-to-Brane ratio displayed in Table 7 [85]. From these, we see that this ratio becomes smaller than unity as soon as one extra dimension is introduced in the theory, decreases further as $n$ takes intermediate values, and increases, while remaining smaller than unity, as $n$ reaches higher (supergravity-inspired) values. Thus, we deduce that, in general, the brane scalar channel is the dominant one, however, for high values of $n$, the bulk emission becomes indeed significant.

The above result gives strong support to the argument presented in [94] where it was argued that most of the energy of a higher-dimensional black hole will be emitted on the brane. The fact that the number of brane-localised degrees of freedom is larger than the bulk ones, combined with the above result that, when both channels are available, the black hole still prefers the brane one, solidifies this argument. However, this matter is far from settled since we have not looked yet at one of the most important species of particles that may be emitted by the black hole into the higher-dimensional spacetime, namely the gravitons. If the probability for graviton emission in the bulk comes out to be much higher than the one for lower-spin fields, then, the bulk-to-brane balance may be overturned.

The graviton equation of motion in the bulk was derived [95] only a few years ago, in the case of a spherically-symmetric, higher-dimensional background. In there, a comprehensive analysis led to Schrödinger-like equations for the three types of gravitational degrees of freedom that one encounters in a higher-dimensional spacetime, namely tensor, vector and scalar ones. In the years that followed, the equations of motion for all three types were studied both analytically [96, 97] and numerically [98, 99]. The analytical approaches led to the derivation of the gravitational radiation spectra either in the intermediate [96] or in the low-energy [97] regime. In the latter case, it was shown [97] that, as long as the energy of the emitted particles remain in the lower part of the spectrum, the total bulk graviton emission rate is sub-dominant to the one for a bulk scalar field, which in turn is subdominant to the one for a brane scalar field. However, a definite answer for the graviton effect on the bulk-to-brane balance can be given only if the complete spectrum for these degrees of freedom is known. This followed from the numerical analysis performed in [98]; according to their results, the energy emission rates for gravitons for the Schwarzschild phase of the black hole behave similarly to the ones for the other degrees of freedom, i.e. they are significantly enhanced as the number of additional 
Table 8 Relative emissivities for brane-localised Standard Model fields and bulk gravitons

\begin{tabular}{ccccccccc}
\hline$n$ & 0 & 1 & 2 & 3 & 4 & 5 & 6 & 7 \\
\hline Scalars & 1 & 1 & 1 & 1 & 1 & 1 & 1 & 1 \\
Fermions & 0.55 & 0.87 & 0.91 & 0.89 & 0.87 & 0.85 & 0.84 & 0.82 \\
G. Bosons & 0.23 & 0.69 & 0.91 & 1.0 & 1.04 & 1.06 & 1.06 & 1.07 \\
Gravitons & 0.053 & 0.61 & 1.5 & 2.7 & 4.8 & 8.8 & 17.7 & 34.7 \\
\hline
\end{tabular}

spacelike dimensions increases. The exact enhancement factors in terms of $n$ appear in the last row of Table 5 and a direct comparison is possible: clearly, the bulk graviton emission rate is the one that exhibits the biggest in magnitude enhancement factor.

Similar results follow when the relative emissivities are computed - these are displayed in Table 8 Due to the aforementioned enhancement factor, the gravitons, from an insignificant part of the total emission in 4 dimensions, become the dominant type of particles emitted by the black hole as soon as $n \geq 2$. How does this affect the bulk-to-brane energy balance then? Surprisingly, it is not in a position to overturn the dominance of the brane channel. The reason for this is that in the relative emissivities for gravitons displayed in Table 8 the total number of gravitational degrees of freedom has already been taken into account. On the other hand, the relative emissivities for the SM fields correspond to individual scalar, fermionic and gauge bosonic degrees of freedoms. When the total number of SM degrees of freedom (not to mention the beyond-the-SM ones) living on the brane is included in the calculation of the total "brane emissivity", the brane channel turns out to be the dominant one once again 6 .

Have we therefore settled the question of the brane-to-bulk energy balance? Perhaps, not. The discussion up to now referred to the Schwarzschild phase in the life of the black hole, and another study needs to be performed for the spin-down phase. The only results available in the literature for the brane-to-bulk ratio in the case of a higher-dimensional, rotating black-hole background, are the analytic ones for scalar fields presented in [102]. In Fig. 11, we display the ratio of the differential energy emission rate for scalar fields living on the brane over the one for bulk scalar fields from a higher-dimensional black hole with line-element given by Eq. 43). From the left plot of Fig. 11, we see that for a black hole with fixed angular momentum $\left(a_{*}=0.5\right)$ the brane-to-bulk ratio remains above unity for all values of $n$. On the other hand, from the right plot we observe that, for a 5-dimensional black hole, the same ratio is again larger than unity but it decreases as either the angular momentum of the black hole or the energy of the particle increases. It would be indeed interest-

\footnotetext{
${ }^{6}$ We note that in the presence of higher-derivative curvature terms in the theory, such as the GaussBonnet term, it has been found [100] that the bulk emission might become the dominant one for specific values of the black hole mass and Gauss-Bonnet coupling constant even for the sphericallysymmetric Schwarzschild phase. Also, in the case that the model allows for fermions to propagate in the bulk, the bulk-to-brane ratio in the fermionic channel exceeds unity even by an order of magnitude [101].
} 


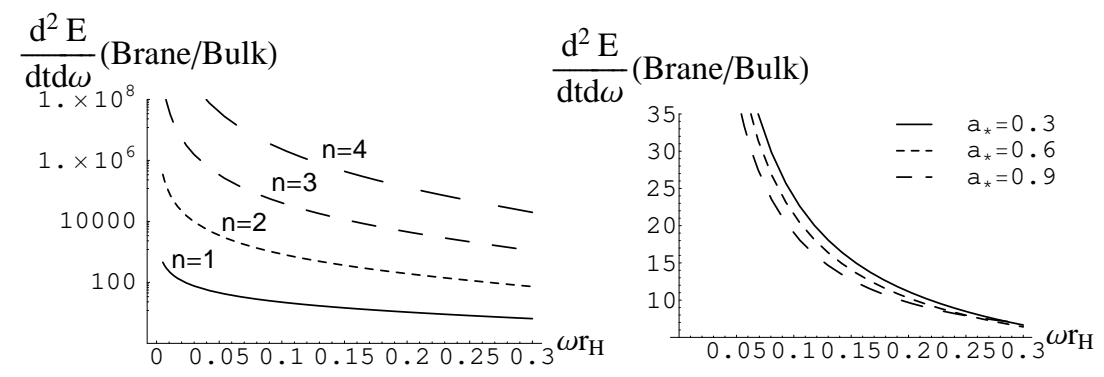

Fig. 11 Brane-to-Bulk ratio of the differential energy emission rates for scalar fields during the spin-down phase in terms of $n$ (left plot) and $a$ (right plot).

ing to check whether the brane dominance in the emission of scalar fields persists over the whole frequency regime, especially for large values of $a$ [103].

\subsection{Deducing Basic Information}

Let us now discuss the methodology one should follow, in case we witness the creation of miniature black holes in a collider experiment. In order to deduce any useful information on the fundamental, higher-dimensional theory, we need to compute with the greatest possible accuracy two quantities: the mass of the black hole and its temperature.

During a high-energy collision of composite particles, it is impossible to know which pair of partons led to the creation of the black hole and what was its total energy. Further losses of energy in the form of gravitational or visible radiation during the balding phase complicates things even more. The black-hole mass can therefore be reconstructed only through the measurement of the energy of the particles that appear in the final state after the evaporation of the black hole [64]. Clearly, any missing energy will greatly reduce the efficiency of the method, therefore one needs to focus on events with little or no missing energy. To this end, a cut is imposed on events with missing energy $E>100 \mathrm{GeV}$, so that the black hole mass resolution is about $4 \%$, i.e. $\pm 200 \mathrm{GeV}$ if $M_{B H}=5 \mathrm{TeV}$ [104].

The temperature of the black hole can be determined by performing a fit on the detected Hawking radiation spectra [64]. Preferably, these spectra should come from events involving only photons and electrons in the final state. The reason is that (a) these events would have a very low background, and (b) the energy resolution of these particles is excellent even at high energies.

Once the temperature $T_{H}$ and mass $M_{B H}$ of the black hole are found with the greatest possible accuracy, one could proceed to determine the dimensionality of spacetime, in other words the value of $n$. From the temperature-horizon radius relation [18, we may write [64 

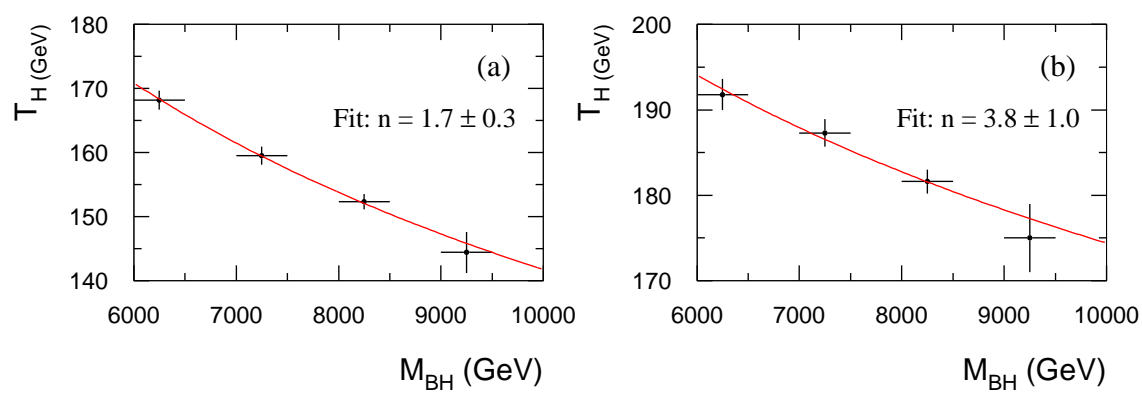

Fig. 12 Plots relating the black-hole mass and temperature measurements, and the derived value of $n$, for constant (left plot) and variable (right plot) temperature [104].

$$
\log \left(T_{H}\right)=-\frac{1}{n+1} \log \left(M_{B H}\right)+\text { const }
$$

Then, the value of $n$ can simply follow by determining the slope of the straightline fit of the data relating $M_{B H}$ and $T_{H}$. The above method is naturally not free of problems - indicatively, we may mention the following:

- The resolution in the measurement of the black-hole mass $M_{B H}$ may not be good

- The black hole temperature $T_{H}$ changes (increases) as a function of time as the evaporation progresses

- The multiplicity of particles in the final state of the evaporation decreases for high values of $n$

- Secondary particles that do not come directly from the evaporating black hole may obscure the spectrum

We have already discussed the first problem associated with the determination of the black-hole mass. Let us briefly discuss the second one involving the temperature. We consider the special case with fundamental Planck scale given by $M_{*}=1 \mathrm{TeV}$ and number of additional dimensions $n=2$. We will pretend that we do not know the value of $n$ but rather we are trying to find it through Eq. (54). We can assume that the temperature of the black hole either remains constant or it increases as the time goes by. Then, the use of Eq. (54) leads to the two plots, respectively, appearing in Fig. 12 [104]. As we see, by fitting the slope of the straight line, we obtain $n=1.7 \pm 0.3$ in the first case, and $n=3.8 \pm 1.0$ in the second. A realistic model should be in a position to take into account that the temperature of the black hole is indeed increasing as the evaporation progresses but also that the lifetime of the black hole is extremely short. As a result, the real situation should actually be somewhere in between the two cases considered above, and an accurate fitting should be in a position to produce the correct value of $n$ which lies indeed between the two derived values.

The multiplicity of particles emitted by the evaporating black hole depends strongly on the black hole mass and its temperature - roughly, the first quantity 
stands for the amount of energy available for emission and the second for the average energy that each emitted particle carries away. More accurately, the number of particles emitted by the black hole is given by the relation [64]

$$
\langle N\rangle=\left\langle\frac{M_{B H}}{E}\right\rangle \simeq \frac{M_{B H}}{2 T_{H}} .
$$

If, given the extremely short lifetime of the black hole, we assume that its mass remains constant and that the black hole evaporates instantly into a number of particles, the multiplicity then depends on the value of $T_{H}$. From the entries of Table 4. we see that the value of the temperature increases as the number of additional dimensions $n$ increases, too. In Fig. 13, we display a plot [104] showing the multiplicity of particles emitted from a black hole as a function of $M_{B H}$, and for various values of $n$ [increasing from 2 (top) to 6 (bottom)] for $M_{*}=1 \mathrm{TeV}$. From this, it is clear that, while for small values of $n$, a black hole, that might be created at the LHC, can emit up to 25 particles, for large values of $n$, this number drops at around 10. As a result, the number of data points that we need to construct the $T_{H}-M_{B H}$ line reduces significantly with $n$, and with it the accuracy in the determination of its slope. While therefore, by using Eq. (54), we might be in a position to obtain a rather accurate value of $n$ if that lies in the lower part of its range, it might be very difficult to distinguish between the cases with $n=5, n=6$ or $n=7$.

Many experiments, looking for beyond the SM physics, have included searches for extra dimensions and miniature black holes in their research programs. At the Large Hadron Collider alone, three collaborations (ALICE, ATLAS and CMS) are planning to do so. But what type of particles and signatures should we expect to see in the detectors? Will we be able to see the Hawking radiation emission spectra that we presented in the previous sections for elementary SM degrees of freedom (the so-called "primary" particles), or maybe "secondary" composite particles will be detected instead? In order to have a better understanding of the type of particles expected to be seen in the final state, we need a Black Hole Event Generator (BHEG) that simulates the black-hole production and decay process given a number of initial conditions. The method followed in a BHEG is roughly the following:

Fig. 13 Multiplicity of particles emitted by a black hole as the number of the additional spacelike dimensions $n$ increases from 2 (top curve) to 6 (bottom curve) [104].

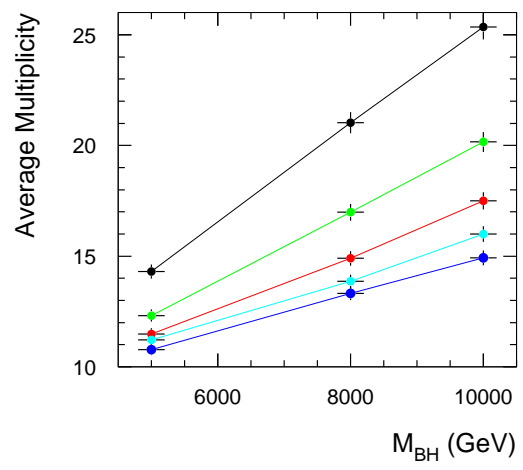


Table 9 Predictions for the relative emissivities of SM fields [104] derived by CHARYBDIS

\begin{tabular}{ccccccccc}
\hline Type & Quarks & Gluons & Charged leptons & Neutrinos & Photons & $Z^{0}$ & $W^{ \pm}$ & Higgs \\
\hline$(\%)$ & 63.9 & 11.7 & 9.4 & 5.1 & 1.5 & 2.6 & 4.7 & 1.1 \\
\hline
\end{tabular}

- For a given center-of-mass energy $E$ of the colliding particles, the black-hole mass $M_{B H}$ is estimated as a fraction of $E$

- The theoretically predicted emission rates for the "primary" particles are fed to the BHEG and the "secondary" particle spectra are produced

At the moment, there are several Black Hole Event Generators that have been constructed: CHARYBDIS [105], Catfish [106] and TRUENOIR [107]. For example, the CHARYBDIS generator uses the HERWIG program [108] to handle all the QCD interactions, hadronization and secondary decays. It also makes specific predictions for the relative emissivities of the different species of SM particles expected to be detected. These are shown in Table 9 [104] from where we easily deduce that the dominant type of elementary particles emitted by the black hole should be the quarks. The exact spectrum of emitted particles depends also on what happens during the final phase in the life of the black hole, i.e. whether the black hole evaporates completely by emitting a few energetic particles or a stable remnant is formed [109, 110, 111, 112, 113, 114]. For this reason, BHEG's are equipped with an option regarding the nature of the final state of the black hole that can be changed at will leading each time to the corresponding radiation spectra. Finally, any observed deviations from the anticipated behaviour stemming from standard QCD could be considered as additional observable signatures of the black-hole formation. For instance, QCD events with high transverse momentum are expected to become gradually more rare as the energy of the collision increases [27]; on the contrary, black hole events with high transverse momentum dominate over the QCD events with this happening at lower energies the smaller the fundamental gravity scale $M_{*}$ is [115]. In addition, in a standard QCD process, one would expect to see the typical back-to-back di-jet production seen in $p+p$ collisions with a particle distribution peaked at $\Delta \phi=0$ and $\pi$, with $\Delta \phi$ being the diference in the azimuthal coordinate between the two emitted hadrons; as the black hole decays through the emission of individual, sequential "primary" particles that lead to mono-jet events, we expect the back-to-back di-jets to be strongly suppressed in the case of the black-hole formation [115, 116].

If we, therefore, wished to summarise some of the most interesting phenomena associated with the existence of a low-scale gravity and the production of higherdimensional black holes, we should mention the following (see [117], for a complementary discussion on this):

- Large cross-sections, that increase with the center-of-mass energy of the collision unlike every other SM process - in such a case, the accurate measurement of the cross-section could lead to the value of $M_{*}$ 
- Primary particles emitted by the black hole with a thermal spectrum and a much higher multiplicity than any other SM process

- Energy emission rates and relative emissivities for different species of fields determined by the number of additional spacelike dimensions

- Non-trivial angular distribution in the radiation spectra coming from the spindown phase

- Comparison of the observed with the predicted spectra could lead to the detection of the final remnant - its presence would increase the multiplicity of particles in the final decay, lower the total transverse momentum by an amount equal to its mass, and, if charged, it could even be directly detected via an ionizing track in the detector

- Events with high transverse momentum, above the expected QCD background

- Strong suppression of back-to-back di-jet events contrary to the expected QCD behaviour

- A significant amount of missing energy - larger than the one for SM or SUSY due to the emission of weakly interacting particles on the branes and of gravitons or scalars in the bulk.

\subsection{Schwarzschild - de Sitter Black Holes}

We would like to finish the discussion of the properties and fate of higher-dimensional black holes with a brief reference to the class of black holes that are formed in the presence of a positive cosmological constant $\Lambda$ in the higher-dimensional spacetime. The geometrical background around such a Schwarzschild - de Sitter black hole is given by the line element [30]

$$
d s^{2}=-h(r) d t^{2}+\frac{d r^{2}}{h(r)}+r^{2} d \Omega_{2+n}^{2}
$$

where

$$
h(r)=1-\frac{\mu}{r^{n+1}}-\frac{2 \kappa_{D}^{2} \Lambda r^{2}}{(n+3)(n+2)},
$$

with $\kappa_{D}^{2} \equiv 8 \pi G_{D}=8 \pi / M_{*}^{2+n}$ and $\mu$ given again by Eq. (45). The equation $h(r)=$ 0 has two real, positive solutions, $r_{H}$ and $r_{C}$ standing for the black hole and the cosmological horizon, respectively. The temperature of the black hole is given by the expression [118]

$$
T_{H}=\frac{1}{\sqrt{h\left(r_{0}\right)}} \frac{1}{4 \pi r_{H}}\left[(n+1)-\frac{2 \kappa_{D}^{2} \Lambda}{(n+2)} r_{H}^{2}\right]
$$

where $r_{0}$ is the value of the radial coordinate where the metric function $h(r)$ reaches its maximum value - the presence of the factor $1 / \sqrt{h\left(r_{0}\right)}$ in the expression for the temperature is necessary for its consistent definition [120]. A similar expression can 


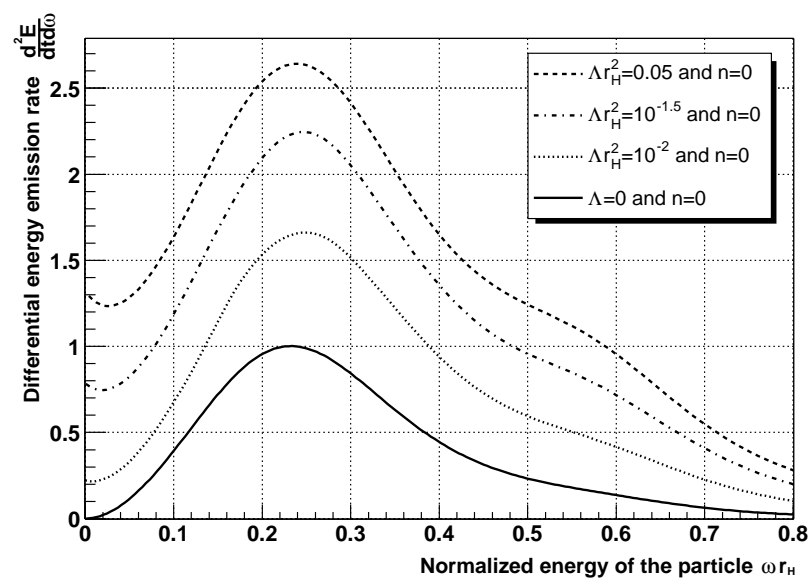

Fig. 14 Energy emission rate for a 4-dimensional black hole in terms of the cosmological constant $\Lambda$.

be written for the temperature $T_{C}$ corresponding to the cosmological horizon - the fact that $r_{C}>r_{H}$ guarantees that $T_{C}<T_{H}$, therefore the flow of energy is from the black hole towards the remaining spacetime.

The line-element of the gravitational background on the brane follows as before by fixing the values of the additional angular coordinates to $\theta_{i}=\pi / 2$. It is then straightforward to write the equation of motion of a scalar field propagating in the projected-to-the-brane background. By solving the radial part of the equation of motion, we may again determine the absorption probability $|\mathscr{A}(\omega)|^{2}$, and in turn the energy emission rate. As in the case of a flat spacetime, the energy emission rate is found to be greatly enhanced with the number of extra dimensions $n$ but also with the value of the cosmological constant $\Lambda$. This enhancement is clearly shown in Fig. 14 [118. What is, however, more important is the fact that, unlike in the case where $\Lambda=0$, for $\Lambda \neq 0$, the emission curve reaches an asymptotic non-zero value as $\omega \rightarrow 0$. This asymptotic value increases with the value of $\Lambda$ and it might, in principle, be used to "read" the value of the cosmological constant from the observed radiation spectra. This non-zero asymptotic value is due to the fact that, unlike in the case of a flat spacetime, the absorption probability acquires a non-vanishing value when $\omega \rightarrow 0$. This is given by the expression [118] (see also [119])

$$
|\mathscr{A}(\omega=0)|^{2}=\frac{4 r_{C}^{2} r_{H}^{2}}{\left(r_{C}^{2}+r_{H}^{2}\right)^{2}},
$$

and is clearly caused by the presence of the cosmological horizon in the theory - in the limit $r_{C} \rightarrow \infty$, the asymptotic value of the absorption probability, and in turn of the energy emission rate, reduces to zero. This effect is independent of the existence of additional spacelike dimensions and should be manifest also in the radiation spectra of 4-dimensional primordial black holes. 


\section{Conclusions}

In the context of the theories predicting the existence of either Large or Warped Extra Dimensions, a low-scale gravitational theory, characterised by a fundamental Planck scale $M_{*}$ much smaller than the 4-dimensional one $M_{P}$, can be realised. This theory becomes accessible as soon as the energy of a given experiment exceeds $M_{*}$, and manifests itself through a number of strong gravity effects. These effects should be present even at ordinary Standard Model particle collisions, taking place, for instance, at ground-based colliders. As a matter of fact, it is expected that during collisions with $E>M_{*}$ we should witness the creation of not point-like particles anymore but of extended heavy objects. One such type of objects are black holes, one of the most fascinating classes of solutions in General Relativity.

The Large Hadron Collider at CERN will have a center-of-mass energy of 14 $\mathrm{TeV}$, i.e. more than an order of magnitude larger than the value of the fundamental Planck scale $M_{*}=1 \mathrm{TeV}$, suggested by the most optimistic scenaria with extra dimensions. It becomes then a natural place to look for strong gravity effects, and possibly for the creation of black holes. Studies have shown that their production can be realised as long as the energy of the collision exceeds at least the value of 8 $\mathrm{TeV}$. At the same time, the produced black holes are expected to have a mass of at least a few times the value of the fundamental Planck scale if we want the classical theory of General Relativity and its predictions to be still applicable. According to the above restrictions, the Large Hadron Collider is found to lie on the edge of both the classical regime and of the black hole creation threshold.

The calculation of the value of the corresponding production cross-section has attracted a great attention over the years. The current results seem to support the claim that this value is significant and that it will lead to the creation of, at least, a few black-hole events per day. In addition, the study of the properties of these higherdimensional black holes suggest that the presence of extra dimensions greatly facilitates their creation: for instance, the horizon radius of these black holes, although tiny, is orders of magnitude larger compared to the one for a 4-dimensional black hole with the same mass.

When it comes to the detection of these events, the terms are also favourable. The most important observable associated with the creation of the black hole will be the emission of Hawking radiation, in the form of elementary particles, as the black hole evaporates. The corresponding radiation spectrum will be centered around the value of the temperature of the black hole, which, for the mass values that would allow their creation at LHC, comes out to be in the range of $100-600 \mathrm{GeV}$. A thorough theoretical study, employing either analytic or numerical techniques, is necessary in order to determine, and thus predict, the exact Hawking radiation spectrum from a decaying black hole. The differential energy emission rates are found to depend on a number of particle and spacetime properties, and thus to encode a valuable amount of information for the gravitational background and for the species of particles emitted. Some of the quantities on which we may deduce information are the number of additional dimensions that exist transversely to the brane, the black-hole angular momentum, the cosmological constant, the spin of the emitted particles, and so on. 
In order to make a realistic prediction of the radiation spectra and also to model in more detail the dynamical aspects of the production and evaporation process, Black Hole Event Generators have been constructed. The exact form of the radiation spectra, together with an additional number of distinct observable signatures should make the detection of Black Holes, and thus of the existence itself of the extra dimensions, possible at the Large Hadron Collider. Hopefully, during the coming years, our understanding of particle and gravitational physics, and of the fundamental theory that describes them, will be considerably extended beyond the current limits.

Acknowledgements I am grateful to my collaborators (J. March-Russell, C. Harris, A. Barrau, J. Grain, G. Duffy, E. Winstanley, M. Casals, S. Creek, O. Efthimiou, K. Tamvakis and S. Dolan, in chronological order) for their valuable help and inspiration while trying to uncover some of the secrets of the higher-dimensional black holes. I would also like to thank the organisers of the 4th Aegean Summer School on Black holes for their kind invitation to present these lectures. I finally acknowledge financial support from the UK PPARC Research Grant PPA/A/S/2002/00350 and participation in the RTN Universenet (MRTN-CT-2006035863-1 and MRTN-CT-2004-503369).

\section{References}

1. I. Antoniadis, Phys. Lett. B 246, 377 (1990).

2. P. Horava and E. Witten, Nucl. Phys. B 460, 506 (1996); Nucl. Phys. B 475, 94 (1996).

3. J. Lykken, Phys. Rev. D 54, 3693 (1996).

4. K. Akama, Lect. Notes Phys. 176, 267 (1982).

5. V. A. Rubakov and M. E. Shaposhnikov, Phys. Lett. B 125, 139 (1983); Phys. Lett. B 125, 136 (1983).

6. M. Visser, Phys. Lett. B 159, 22 (1985).

7. G. W. Gibbons and D. L. Wiltshire, Nucl. Phys. B 287, 717 (1987).

8. N. Arkani-Hamed, S. Dimopoulos and G. Dvali, Phys. Lett. B 429, 263 (1998); Phys. Rev. D 59, 086004 (1999).

9. I. Antoniadis, N. Arkani-Hamed, S. Dimopoulos and G. R. Dvali, Phys. Lett. B 436, 257 (1998).

10. L. Randall and R. Sundrum, Phys. Rev. Lett. 83, 3370 (1999); Phys. Rev. Lett. 83, 4690 (1999).

11. P. Abreu et al. [DELPHI Collaboration], Eur. Phys. J. C17, 53 (2000).

12. G. Abbiendi et al. [OPAL Collaboration], Eur. Phys. J. C18, 253 (2000).

13. D. Acosta et al. [CDF Collaboration], Phys. Rev. Lett. 89, 281801 (2002).

14. C. D. Hoyle, U. Schmidt, B. R. Heckel, E. G. Adelberger, J. H. Gundlach, D. J. Kapner and H. E. Swanson, Phys. Rev. Lett. 86, 1418 (2001).

15. D. J. Kapner, T. S. Cook, E. G. Adelberger, J. H. Gundlach, B. R. Heckel, C. D. Hoyle and H. E. Swanson, Phys. Rev. Lett. 98, 021101 (2007).

16. L. J. Hall and D. R. Smith, Phys. Rev. D60, 085008 (1999).

17. S. Cullen and M. Perelstein, Phys. Rev. Lett. 83, 268 (1999).

18. V. D. Barger, T. Han, C. Kao and R. J. Zhang, Phys. Lett. B461, 34 (1999).

19. C. Hanhart, D. Phillips, S. Reddy and M. Savage, Nucl. Phys. B595, 335 (2001).

20. C. Hanhart, J. A. Pons, D. R. Phillips and S. Reddy, Phys. Lett. B509, 1 (2001).

21. R. Allahverdi, C. Bird, S. Groot Nibbelink and M. Pospelov, Phys. Rev. D69, 045004 (2004).

22. S. Hannestad and G. Raffelt, Phys. Rev. Lett. 87, 051301 (2001).

23. S. Hannestad, Phys. Rev. D64, 023515 (2001). 
24. S. Hannestad and G. Raffelt, Phys. Rev. Lett. 88, 071301 (2002); Phys. Rev. D67, 125008 (2003) [Erratum-ibid. D69, 029901 (2004)].

25. M. Gogberashvili, A.S. Sakharov and E.K.G. Sarkisyan, Phys. Lett. B644, 179 (2007).

26. L. A. Anchordoqui, J. L. Feng, H. Goldberg and A. D. Shapere, Phys. Rev. D68, 104025 (2003).

27. T. Banks and W. Fischler, hep-th/9906038

28. K. S. Thorne, in Magic without Magic, ed. J. R. Klauder (San Fransisco, 1972).

29. D. Ida and K. i. Nakao, Phys. Rev. D 66, 064026 (2002).

30. F. R. Tangherlini, Nuovo Cim. 27, 636 (1963).

31. R. C. Myers and M. J. Perry, Annals Phys. 172, 304 (1986).

32. P. Meade and L. Randall, JHEP 0805, 003 (2008).

33. K.A. Khan and R. Penrose, Nature 229, 185 (1971).

34. P. Szekeres, J. Math. Phys. 13, 286 (1972).

35. T. Dray and G. 't Hooft, Nucl. Phys. B253, 173 (1985); Class. Quant. Grav. 3, 825 (1986).

36. U. Yurtsever, Phys. Rev. D38, 1706 (1988); Phys. Rev. D38, 1731 (1988).

37. P. D. D'Eath and P. N. Payne, Phys. Rev. D46, 658 (1992); Phys. Rev. D46, 675 (1992); Phys. Rev. D46, 694 (1992).

38. G. 't Hooft, Phys. Lett. B198, 61 (1987); Nucl. Phys. B304, 867 (1988); Nucl. Phys. B335, 138 (1990).

39. S. B. Giddings and M. Srednicki, Phys. Rev. D 77, 085025 (2008).

40. D. J. Gross and P. F. Mende, Phys. Lett. B197, 129 (1987); Nucl. Phys. B303, 407 (1988).

41. D. Amati, M. Ciafaloni and G. Veneziano, Phys. Lett. B197, 81 (1987); Int. J. Mod. Phys. A3, 1615 (1988); Phys. Lett. B216, 41 (1989).

42. S. B. Giddings, D. J. Gross and A. Maharana, Phys. Rev. D 77, 046001 (2008).

43. H. Verlinde and E. Verlinde, Nucl. Phys. B371, 246 (1992).

44. P.C. Aichelburg and R.U. Sexl, Gen. Rel. Grav. 2, 303 (1971).

45. V. P. Frolov and I. D. Novikov, "Black hole physics: Basic concepts and new developments," Kluwer Academic (Dordrecht, Netherlands, 1998).

46. R. Penrose, presented at the Cambridge University Seminar, unpublished (1974).

47. V. Cardoso and J. P. S. Lemos, Phys. Lett. B538, 1 (2002); Phys. Rev. D67, 084005 (2003).

48. U. Sperhake, V. Cardoso, F. Pretorius, E. Berti and J.A. Gonzalez, arXiv:0806.1738 [gr-qc].

49. D. M. Eardley and S. B. Giddings, Phys. Rev. D 66, 044011 (2002).

50. H. Yoshino and Y. Nambu, Phys. Rev. D66, 065004 (2002); Phys. Rev. D 67, 024009 (2003).

51. M. B. Voloshin, Phys. Lett. B518, 137 (2001); Phys. Lett. B524, 376 (2002).

52. S. Dimopoulos and R. Emparan, Phys. Lett. B526, 393 (2002).

53. E. Kohlprath and G. Veneziano, JHEP 0206, 057 (2002).

54. S. N. Solodukhin, Phys. Lett. B533, 153 (2002).

55. T. G. Rizzo, JHEP 0202, 011 (2002).

56. S. D. H. Hsu, Phys. Lett. B555, 92 (2003).

57. H. Yoshino and V. S. Rychkov, Phys. Rev. D71, 104028 (2005).

58. M. Cavaglia, S. Das and R. Maartens, Class. Quant. Grav. 20, L205 (2003).

59. H. Yoshino and R. B. Mann, Phys. Rev. D74, 044003 (2006).

60. D. Ida, K. y. Oda and S. C. Park, Phys. Rev. D 67, 064025 (2003) [Erratum-ibid. D 69, 049901 (2004)].

61. B. Koch, Phys. Lett. B663/4, 334 (2008).

62. S. B. Giddings, Phys. Rev. D 67, 126001 (2003).

63. S. B. Giddings and S. Thomas, Phys. Rev. D 65, 056010 (2002).

64. S. Dimopoulos and G. Landsberg, Phys. Rev. D 87, 161602 (2001).

65. P. C. Argyres, S. Dimopoulos and J. March-Russell, Phys. Lett. B 441, 96 (1998).

66. P. Kanti, Int. J. Mod. Phys. A 19, 4899 (2004).

67. S. W. Hawking, Commun. Math. Phys. 43, 199 (1975).

68. W. G. Unruh, Phys. Rev. D 10, 3194 (1974); Phys. Rev. D 14, 3251 (1976).

69. A. C. Ottewill and E. Winstanley, Phys. Rev. D 62, 084018 (2000).

70. Y.B. Zel'dovich, JETP Lett. 14, 180 (1971).

71. P. Kanti and J. March-Russell, Phys. Rev. D 66, 024023 (2002). 
72. P. Kanti and J. March-Russell, Phys. Rev. D D67, 104019 (2003).

73. V. Frolov and D. Stojkovic, Phys. Rev. D 67, 084004 (2003).

74. C. M. Harris and P. Kanti, Phys. Lett. B 633, 106 (2006).

75. G. Duffy, C. Harris, P. Kanti and E. Winstanley, JHEP 0509, 049 (2005).

76. D. Ida, K. y. Oda and S. C. Park, Phys. Rev. D 71, 124039 (2005).

77. M. Casals, P. Kanti and E. Winstanley, JHEP 0602, 051 (2006).

78. D. Ida, K. y. Oda and S. C. Park, Phys. Rev. D 73, 124022 (2006).

79. M. Casals, S. R. Dolan, P. Kanti and E. Winstanley, JHEP 0703, 019 (2007).

80. E. Newman and R. Penrose, J. Math. Phys. 3, 566 (1962).

81. S. Chandrasekhar, The Mathematical Theory of Black Holes (Oxford University Press, New York, 1983).

82. J. N. Goldberg, A. J. MacFarlane, E. T. Newman, F. Rohrlich and E. C. Sudarshan, J. Math. Phys. 8, 2155 (1967).

83. S. A. Teukolsky, Phys. Rev. Lett. 29, 1114 (1972); Astrophys. J. 185, 635 (1973).

84. S. S. Gubser, I. R. Klebanov and A. A. Tseytlin, Nucl. Phys. B499, 217 (1997).

85. C. M. Harris and P. Kanti, JHEP 0310, 014 (2003).

86. D. N. Page, Phys. Rev. D 13, 198 (1976).

87. C. Flammer, Spheroidal Wave Functions (Stanford University Press, Stanford, USA, 1957).

88. A. A. Starobinskii and S. M. Churilov, Sov. Phys.-JETP 38, 1 (1974).

89. E. D. Fackerell and R. G. Crossman, J. Math. Phys. 18, 1849 (1977).

90. E. Seidel, Class. Quant. Grav. 6, 1057 (1989).

91. S. Creek, O. Efthimiou, P. Kanti and K. Tamvakis, Phys. Rev. D 75, 084043 (2007).

92. S. Creek, O. Efthimiou, P. Kanti and K. Tamvakis, Phys. Rev. D 76, 104013 (2007).

93. C. Muller, in Lecture Notes in Mathematics: Spherical Harmonics (Springer-Verlag, BerlinHeidelberg, 1966).

94. R. Emparan, G. T. Horowitz and R. C. Myers, Phys. Rev. Lett. 85, 499 (2000).

95. H. Kodama and A. Ishibashi, Prog. Theor. Phys. 110, 701 (2003); Prog. Theor. Phys. 111, 29 (2004).

96. A. S. Cornell, W. Naylor and M. Sasaki, JHEP 0602, 012 (2006).

97. S. Creek, O. Efthimiou, P. Kanti and K. Tamvakis, Phys. Lett. B 635, 39 (2006).

98. V. Cardoso, M. Cavaglia and L. Gualtieri, Phys. Rev. Lett. 96, 071301 (2006) [Erratum-ibid. 96, 219902 (2006)]; JHEP 0602, 021 (2006).

99. D. K. Park, Phys. Lett. B 638, 246 (2006).

100. J. Grain, A. Barrau and P. Kanti, Phys. Rev. D 72, 104016 (2005).

101. H. T. Cho, A. S. Cornell, J. Doukas and W. Naylor, Phys. Rev. D 77, 016004 (2008).

102. S. Creek, O. Efthimiou, P. Kanti and K. Tamvakis, Phys. Lett. B 656, 102 (2007).

103. M. Casals, S. R. Dolan, P. Kanti and E. Winstanley, JHEP 0806, 071 (2008).

104. C. M. Harris, M. J. Palmer, M. A. Parker, P. Richardson, A. Sabetfakhri and B. R. Webber, JHEP 0505, 053 (2005).

105. C. M. Harris, P. Richardson and B. R. Webber, JHEP 0308, 033 (2003).

106. M. Cavaglia, R. Godang, L. Cremaldi and D. Summers, Comput. Phys. Commun. 177, 506 (2007).

107. G. L. Landsberg, J. Phys. G 32, R337 (2006).

108. G. Corcella et al., JHEP 0101, 010 (2001).

109. Y. Aharonov, A. Casher and S. Nussinov, Phys. Lett. B 191, 51 (1987).

110. T. Banks, A. Dabholkar, M. R. Douglas and M. O’Loughlin, Phys. Rev. D 45, 3607 (1992).

111. T. Banks and M. O'Loughlin, Phys. Rev. D 47, 540 (1993).

112. T. Banks, M. O'Loughlin and A. Strominger, Phys. Rev. D 47, 4476 (1993).

113. S. B. Giddings, Phys. Rev. D 49, 947 (1994).

114. B. Koch, M. Bleicher and S. Hossenfelder, JHEP 0510, 053 (2005).

115. T. J. Humanic, B. Koch and H. Stoecker, Int. J. Mod. Phys. E 16, 841 (2007).

116. B. Koch, M. Bleicher and H. Stoecker, J. Phys. G 34, S535 (2007).

117. S. B. Giddings, AIP Conf. Proc. 957, 69 (2007).

118. P. Kanti, J. Grain and A. Barrau, Phys. Rev. D 71, 104002 (2005).

119. T. Harmark, J. Natario and R. Schiappa, arXiv:0708.0017 [hep-th].

120. R. Bousso and S. W. Hawking, Phys. Rev. D 54, 6312 (1996). 